\title{
Irreversible Qubit-Photon Coupling for the Detection of Itinerant Microwave Photons
}

\author{
Raphaël Lescanne, ${ }^{1,2}$ Samuel Deléglise, ${ }^{3}$ Emanuele Albertinale ${ }^{5}$ Ulysse Réglade, ${ }^{4,1}$ Thibault Capelle, ${ }^{3}$ \\ Edouard Ivanov, ${ }^{3}$ Thibaut Jacqmin, ${ }^{3}$ Zaki Leghtas, ${ }^{4,1,2}$ and Emmanuel Flurin $\circledast^{5,3,1, *}$ \\ ${ }^{1}$ Laboratoire de Physique de l'Ecole Normale Supérieure, ENS, Université PSL, \\ CNRS, Sorbonne Université, Université Paris-Diderot, Sorbonne Paris Cité, F-75005 Paris, France \\ ${ }^{2}$ QUANTIC team, INRIA de Paris, 2 Rue Simone Iff, 75012 Paris, France \\ ${ }^{3}$ Laboratoire Kastler Brossel, Sorbonne Université, CNRS, ENS-PSL Research University, \\ Collège de France, 4 place Jussieu, F-75252 Paris, France \\ ${ }^{4}$ Centre Automatique et Systèmes, Mines-ParisTech, PSL Research University, \\ 60, bd Saint-Michel, 75006 Paris, France \\ ${ }^{5}$ Quantronics group, SPEC, CEA, CNRS, Université Paris-Saclay, \\ CEA Saclay 91191 Gif-sur-Yvette Cedex, France
}

(Received 20 May 2019; accepted 7 April 2020; published 18 May 2020)

\begin{abstract}
Single photon detection is a key resource for sensing at the quantum limit and the enabling technology for measurement-based quantum computing. Photon detection at optical frequencies relies on irreversible photoassisted ionization of various natural materials. However, microwave photons have energies 5 orders of magnitude lower than optical photons, and are therefore ineffective at triggering measurable phenomena at macroscopic scales. Here, we report the observation of a new type of interaction between a single twolevel system (qubit) and a microwave resonator. These two quantum systems do not interact coherently; instead, they share a common dissipative mechanism to a cold bath: the qubit irreversibly switches to its excited state if and only if a photon enters the resonator. We have used this highly correlated dissipation mechanism to detect itinerant photons impinging on the resonator. This scheme does not require any prior knowledge of the photon waveform nor its arrival time, and dominant decoherence mechanisms do not trigger spurious detection events (dark counts). We demonstrate a detection efficiency of $58 \%$ and a record low dark count rate of 1.4 per millisecond. This work establishes engineered nonlinear dissipation as a key enabling resource for a new class of low-noise nonlinear microwave detectors.
\end{abstract}

DOI: 10.1103/PhysRevX.10.021038

Subject Areas: Quantum Information

\section{INTRODUCTION}

High-performance photon detectors in the optical domain [1] are the workhorses of various quantum optics experiments: by combining them with Gaussian resources such as optical parametric oscillators or beam splitters, they have been used to generate nonclassical states [2], entanglement between remote stationary qubits [3], long-distance quantum cryptography protocols [4], or one-way quantum computing [5]. In such nondeterministic protocols, the preparation of the desired quantum state is heralded by the detection of a single itinerant photon. Crucially, by conditioning the success of the experiment upon the detection events, such protocols are highly robust against losses and inefficiencies whereas their fidelity is directly linked to

\section{*emmanuel.flurin@cea.fr}

Published by the American Physical Society under the terms of the Creative Commons Attribution 4.0 International license. Further distribution of this work must maintain attribution to the author(s) and the published article's title, journal citation, and DOI. the number of false-positive events, also known as "dark counts" [6]. Transposing such techniques in the microwave domain has been the subject of intense theoretical [7-12] and experimental [13-17] activity as it would leverage the high level of control over superconducting quantum circuits in a modular architecture where various solid-state quantum systems are connected via lossy connection lines [18]. Moreover, such detectors are increasingly sought after due to their applications in the detection of dark matter axions [19], electron-paramagnetic-resonance spectroscopy [20], or quantum-enhanced imaging [21]. In such applications, where the arrival time of the photon is not known in advance, the possibility to operate the detector in a cyclic regime with a short reset time is a crucial requirement.

The detection of individual photons trapped in long-lived microwave cavities has been pioneered by atomic cavity quantum electrodynamics experiments [22,23], and later on transposed to circuit quantum electrodynamics [14,24]. In these detection schemes, the trapping of the photon into a long-lived cavity enhances the interaction to the measurement apparatus. Two fundamental difficulties arise in the detection of itinerant photons: the incoming photon only 
interacts briefly with the detector and it occupies an unknown temporal mode out of a continuum. Recently, several strategies have been explored experimentally. In a first approach, a current biased Josephson circuit develops a macroscopic voltage [13] or a photon avalanche [15] upon the absorption of a single photon. Another strategy involves an ac-modulated flux qubit that transits into an excited state through a photon-induced Raman transition [25]. In this implementation, the switching of the ac modulation induces a significant number of dark counts. Finally, in detection schemes based on the Ramsey interferometry of a transmon qubit, the photon imprints a phase on the qubit as it reflects off a cavity $[16,17]$. These experiments demonstrate the quantum nondemolition (QND) detection of the itinerant photons; however, the dark count rate is directly impacted by the qubit decoherence time.

In the seemingly unrelated area of dissipation engineering [26], one carefully designs the coupling of a quantum system to a bath to achieve desired dissipative dynamics. This approach defeats the natural intuition that quantum systems need to be isolated from their environment. Indeed, the irreversible loss of information toward the bath leads to non-Hermitian dynamics that bring asymptotically the system toward the desired quantum state or manifold. Such a peculiar loss channel can be carefully engineered by parametric interaction mediated by strong microwave pumps. This new paradigm has paved the way toward cavity cooling [27], stabilization of quantum states [28,29] and decoherence-free subspaces for autonomous quantum error corrections [30], and the fabrication of nonreciprocal components that do not rely on an external magnetic field [31,32].

In this work, we use dissipation engineering to perform the quantum nondemolition detection of single itinerant photons by irreversibly coupling the transmission line toward a single two-level system (qubit). A photon propagating in the line is absorbed by the qubit, but the reverse process is inhibited: an excitation in the qubit does not propagate back in the line. Upon arrival of a single photon, the qubit is left in its excited state, leaving ample time for it to be measured with a microwave pulse containing tens to hundreds of photons, which is measurable with readily available amplification techniques [33]. In practice, we demonstrate that implementing this engineered dissipation triggers dark counts at a rate one order of magnitude smaller compared to state-of-the-art experiments [14-17,25].

\section{ENGINEERING THE QUBIT-CAVITY DISSIPATOR}

The detector, depicted in Fig. 1(a), is composed of two superconducting microwave resonators: the buffer, which hosts the incoming field, and the waste, which plays the role of the bath, releases the detected photon. The resonators are coupled through a Josephson junction in a bridge transmon configuration [34], and are strongly coupled to
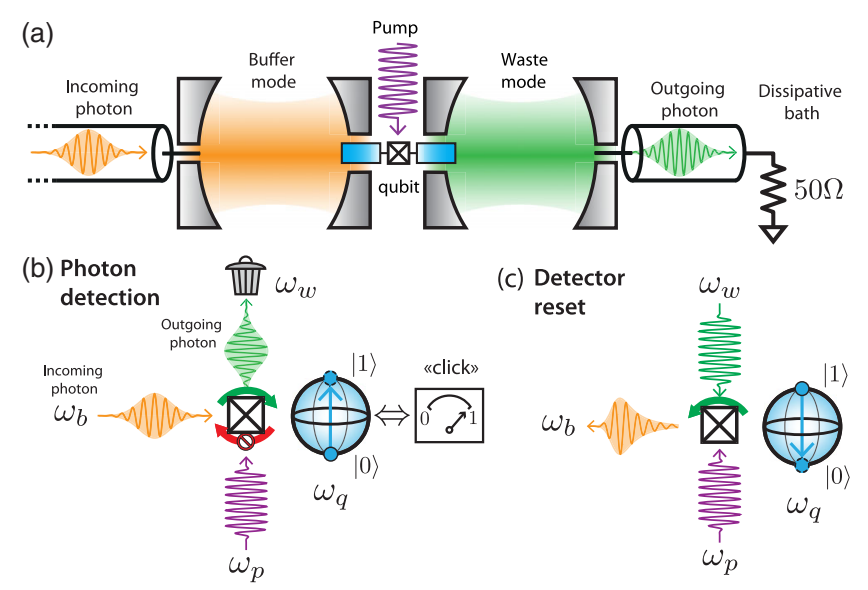

FIG. 1. Principle of the itinerant photon detector. (a) The circuit consists of two microwave modes: a buffer (orange) and a waste (green) coupled to a transmon qubit (blue). Each mode is well coupled to its own transmission line so that a photon can enter the device on the left and leave on the right into the dissipative $50 \Omega$ environment. A pump (purple) is applied on the Josephson junction to make the three-wave mixing interaction resonant [Eq. (2)]. (b) When a photon enters the buffer, the pump converts it via the Josephson nonlinearity (black cross) into one excitation of the qubit and one photon in the waste. The waste excitation is irreversibly radiated away in the transmission line, so that the reverse three-wave process cannot occur. The quantum state of the qubit is measured with a standard dispersive readout via the waste to detect whether or not a photon arrived while the pump was on. (c) When a coherent tone drives the waste, the qubit excitation can combine with waste photons and be released via the Josephson nonlinearity in the buffer, enabling a fast reset of the detector.

transmission lines at a rate $\kappa_{b} / 2 \pi=0.97 \mathrm{MHz}$ and $\kappa_{w} / 2 \pi=2.4 \mathrm{MHz}$, respectively. A microwave drive, referred to as the pump, is applied to the transmon qubit at frequency

$$
\omega_{p}=\bar{\omega}_{q}+\omega_{w}^{e}-\omega_{b}^{g},
$$

where $\bar{\omega}_{q}$ is the qubit frequency shifted by the pump power through the ac-Stark effect (see Appendix G), $\omega_{w}^{e}$ and $\omega_{b}^{g}$ are the buffer and waste frequencies conditioned on the qubit being in its excited state $|e\rangle$ and its ground state $|g\rangle$, respectively. In the absence of the pump $\omega_{q} / 2 \pi=$ $4.532 \mathrm{GHz}, \omega_{b}^{g} / 2 \pi=5.495 \mathrm{GHz}$ and $\omega_{w}^{e} / 2 \pi=5.770 \mathrm{GHz}$. The pumped system is well described by the effective Hamiltonian (see Appendix C),

$$
\hat{H}_{\mathrm{eff}} / \hbar=g_{3} \hat{b} \hat{\sigma}^{\dagger} \hat{w}^{\dagger}+g_{3}^{*} \hat{b}^{\dagger} \hat{\sigma} \hat{w}
$$

where $g_{3}$ is the parametrically activated three-wave mixing rate and verifies $g_{3}=-\xi_{p} \sqrt{\chi_{q b} \chi_{q w}}$. Here, $\chi_{q b} / 2 \pi=1.02 \mathrm{MHz}$ and $\chi_{q w} / 2 \pi=2.73 \mathrm{MHz}$ are the dispersive couplings of the buffer and waste to the qubit, respectively. The pump 
amplitude $\xi_{p}$ is expressed in units of square root of photons and is typically smaller than one. The buffer and waste annihilation operators are denoted $\hat{b}$ and $\hat{w}$, and $\hat{\sigma}$ denotes the lowering operator of the qubit. The itinerant photon to be detected, incident on the buffer, is converted into a pair of excitations in the qubit and in the waste by the term $\hat{b} \hat{\sigma}^{\dagger} \hat{w}^{\dagger}$. We place ourselves in the regime where $\left|g_{3}\right| \ll \kappa_{w}$ so that the photon in the waste is immediately dissipated in the natural environment while the qubit excitation is stored. Since the waste remains close to its vacuum state throughout the dynamics, the reverse process $\left(\hat{b}^{\dagger} \hat{\sigma} \hat{w}\right)$ is effectively inhibited. The subsequent detection of the qubit in the excited state based on single-shot dispersive readout [35] reveals the transit of the photon during the detection time [see Fig. 1(b)].

The irreversible buffer-qubit dynamics, arising from the adiabatic elimination of the waste, is entirely described by a single loss operator as derived in Appendix D

$$
\hat{L}=\sqrt{\kappa_{\mathrm{nl}}} \hat{b} \hat{\sigma}^{\dagger}
$$

where the engineered dissipation rate is $\kappa_{\mathrm{nl}}=4\left|g_{3}\right|^{2} / \kappa_{w}$. This dissipator is unusual for two reasons. First, it is nonlocal [32], since it involves operators from two different modes. Second, it is nonlinear [30], since it involves the product of these operators. These properties are at the heart of the detection process: under the effect of $\hat{L}$, the qubit dissipates toward its excited state conditioned (nonlinear) on the buffer (nonlocal) occupation. As a consequence, the detector is oblivious to the specific mode shape of the incoming photons (within the detector bandwidth) by evacuating the associated entropy into the unread dissipative channel.

\section{PHOTON DETECTOR PERFORMANCES}

\section{A. Efficiency}

The efficiency $\eta$ of the detector is defined as the probability of detecting the qubit in its excited state assuming a single incoming photon. For mode shapes well within the detector bandwidth $\left(\kappa_{\mathrm{nl}}+\kappa_{b}\right) / 2 \pi=1.40 \mathrm{MHz}$ and short compared to the qubit relaxation time, the efficiency is (see Appendix E)

$$
\eta=4 \frac{\kappa_{\mathrm{nl}} \kappa_{b}}{\left(\kappa_{\mathrm{nl}}+\kappa_{b}\right)^{2}} .
$$

The efficiency reaches unity for $\kappa_{\mathrm{nl}}=\kappa_{b}$, when the nonlinear dissipation matches the coupling to the transmission line.

In practice, we satisfy Eq. (1) by performing a calibration experiment [Fig. 2(a)]. The pump power is chosen as the largest that did not induce significant qubit heating, thus maximizing the efficiency to dark count ratio. The chosen pump power leads to $\kappa_{\mathrm{nl}} / 2 \pi=0.43 \mathrm{MHz}$, which results in an efficiency predicted by Eq. (4) of $80 \%$ [red line of
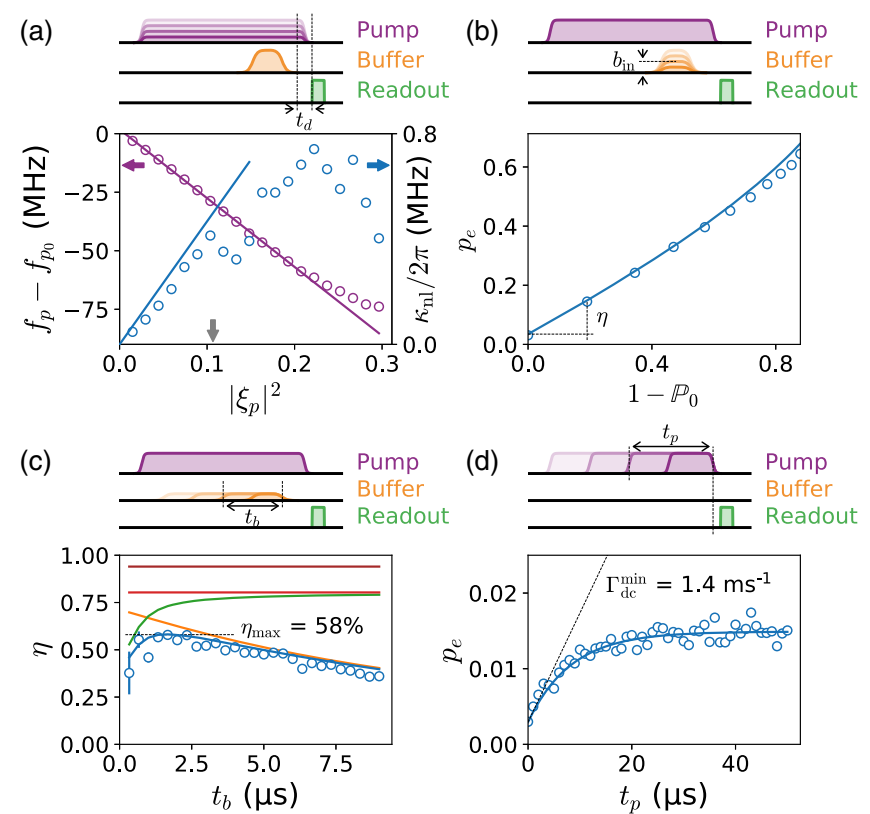

FIG. 2. Calibration and diagnosis of the detector. (a)-(d) A pump tone (purple pulse) of duration $t_{p}$ powers the device through the qubit port, and an incoming coherent wave packet (orange pulse) of duration $t_{b}$ and amplitude $b_{\text {in }}$ is applied on the buffer. The qubit excited state population $p_{e}$ is detected after a delay $t_{d}$ through the waste (green pulse). (a) We plot the pump frequency $f_{p}$ which maximizes $p_{e}$ for various pump powers ( $x$ axis). The first 10 data points (purple open circles) are fitted using a linear regression (purple line), leading to a $y$ intercept within $0.04 \%$ of the expected value $f_{p 0}=\left(\omega_{q}+\omega_{w}^{e}-\omega_{b}^{g}\right) / 2 \pi$. The slope of this line provides a calibration of the pump power in units of photon number $\left|\xi_{p}\right|^{2}$ [see Eq. (C5)]. For each pump power, we plot the induced nonlinear dissipation rate $\kappa_{\mathrm{nl}}$ (blue open circles), which is extracted from a full model simulation [see Eq. (E4)], to best match all measured values of $p_{e}$ for small values of $b_{\text {in }}$, taking into account the readout fidelity (0.94). For pump powers $\left|\xi_{p}\right|^{2}<0.11$, these extracted values follow a linear trend within $10 \%$ of the expected one $\kappa_{\mathrm{nl}}=4\left|\xi_{p}\right|^{2} \chi_{q b} \chi_{w q} / \kappa_{w}$ (blue line). For all subsequent experiments, $\left|\xi_{p}\right|^{2}=0.11$ (gray arrow). The saturation of $\kappa_{\mathrm{nl}}$ is mainly due to the fact that, as $g_{3}$ increases with the pump power, we are leaving the adiabatic elimination regime. (b) Measured $p_{e}$ (open circles) as a function of $1-\mathbb{P}_{0}$, where $\mathbb{P}_{0}$ is the probability of the incoming wave packet being in the vacuum; see Eq. (E13). This quantity is varied by increasing $b_{\text {in }}$ for a fixed $t_{b}=2 \mu \mathrm{s}$. The slope at the origin is the detection efficiency $\eta$, and the $y$ intercept is the dark count. The theory (solid line) is obtained by simulating the full model Eq. (E4). (c) For each $t_{b}$ ( $x$ axis), $\eta$ is measured (open circles) as in (b). The theory curves (solid lines) are obtained by simulating the full model Eq. (E4) with readout infidelity (brown) in four regimes: $T_{1}, \kappa_{b}=\infty$ (red), $\kappa_{b}=\infty$ (orange), $T_{1}=\infty$ (green), and the measured $T_{1}, \kappa_{b}$ (blue). Note that the discrepancy between orange and red at small time is due to the finite switching time of the pump pulse $t_{d}=500 \mathrm{~ns}$. (d) Measured $p_{e}$ (open circles) as a function of $t_{p}$ with $b_{\text {in }}=0$, at a repetition rate of $2 \mathrm{~ms}$. An exponential fit (solid line) rises from the residual excited population after the reset $p_{e}\left(t_{p}=0\right)=0.003$ to the thermal equilibrium $n_{q \text {,th }}=0.015$ at a rate $\Gamma_{\mathrm{dc}}^{\min }=1.4 \mathrm{~ms}^{-1}=$ $n_{q, \mathrm{th}} / T_{1}$ at early time. 
Fig. 2(c)] taking into account the readout fidelity (0.94). The detector efficiency is measured by varying the amplitude of a calibrated coherent pulse [36], as shown in Fig. 2(b). The measurement is repeated for increasing pulse length, as shown in Fig. 2(c). We observe a smooth dependence of the efficiency as a function of the pulse length, that is well reproduced by our model if we take into account the finite detector bandwidth at short pulse duration [green curve of Fig. 2(c)] and the finite qubit lifetime, $T_{1}=8 \mu \mathrm{s}$, for long pulses [orange curve of Fig. 2(c)], resulting in a maximum detection efficiency of $\eta_{\max }=58 \%$ for a $2 \mu$ s pulse length.

\section{B. Dark counts}

A crucial figure of merit of the detector is the dark count rate $\Gamma_{\mathrm{dc}}$, defined as the number of clicks per unit time in the absence of incoming photons. By virtue of our dissipation engineering approach, $\Gamma_{\mathrm{dc}}$ is robust against the two main decoherence mechanisms of the qubit: dephasing and energy relaxation. This is in stark contrast with the best performing schemes relying on Ramsey interferometry $[16,17]$, where the dark count rate scales with the qubit decoherence rate such that $\Gamma_{\mathrm{dc}}^{\text {Ramsey }} \sim 1 /\left(2 T_{2}\right)$; in other words, the detection outcome is blurred after a detection time of order $T_{2}$. In our case, dark counts come from the next limiting process: the qubit thermal excitation. Therefore, the dark count rate is given by the qubit thermalization rate $\Gamma_{\mathrm{dc}} \sim n_{q, \mathrm{th}} / T_{1}$, where $n_{q \text {,th }}$ is the qubit thermal occupancy. Figure 2(d) shows the detection probability $p_{e}$ as a function of the detection window $t_{p}$ in the absence of buffer excitation. At short time compared to the qubit relaxation time $T_{1}$, we find $p_{e}=0.003+\Gamma_{\mathrm{dc}} \times t_{p}$. The first term of 0.003 results from the improper initialization of the qubit in its ground state and detection errors. We find $\Gamma_{\mathrm{dc}}^{\min }=1.4 \mathrm{~ms}^{-1}$, limited by pump-induced heating of the qubit bath [36,37] [see Fig. 2(d)]. We have observed that for the same detection window, when the repetition rate increases from $(2 \mathrm{~ms})^{-1}$ to $(50 \mu \mathrm{s})^{-1}$, the dark count rate increases to $4 \mathrm{~ms}^{-1}$, suggesting that the qubit bath thermalizes over timescales of hundreds of microseconds. In spite of the modest lifetime of our transmon qubit, the dark count rate reported here is one order of magnitude lower than the values reported previously in the literature $[14,16,17,25]$.

\section{Detector reset}

A key requirement for practical photon detectors is a fast and high-fidelity reset, which we achieve using the reverse process $g_{3}^{*} \hat{b}^{\dagger} \hat{\sigma} \hat{w}$ [see Fig. 1(c)]. By shining a resonant tone on the waste in the presence of the pump, the qubit relaxes to the ground state with the emission of a photon in the buffer, leading to a fast reset at a rate $\gamma_{\text {reset }}=(370 \mathrm{~ns})^{-1}$ and a ground state preparation fidelity of $F_{|g\rangle}^{\text {reset }}=99.3 \%$ improved to $F_{|g\rangle}^{\text {herald }}=99.6 \%$ with a subsequent heralding
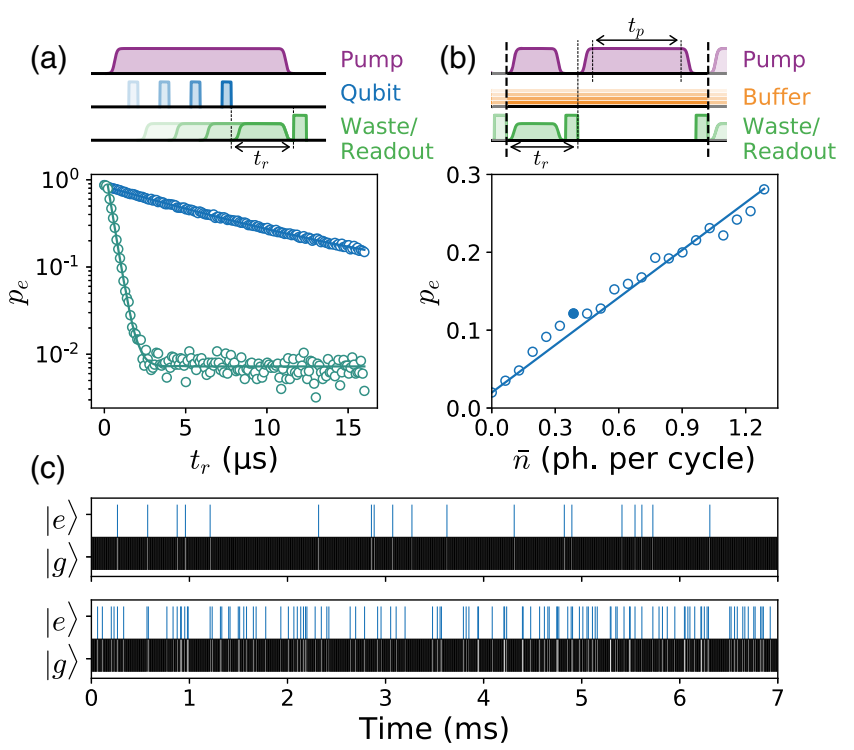

FIG. 3. Reset protocol and cyclic operation. (a) The qubit is initialized in its excited state (blue pulse) and the pump is activated. The decay of $p_{e}$ is measured (open circles) in the presence (green) or absence (blue) of a reset tone of duration $t_{r}$ applied to the waste mode (green pulse). The exponential fits (solid lines) yield a qubit $T_{1}$ reduced from $7.7 \mu$ s to $370 \mathrm{~ns}$. Note that the residual qubit population is reduced in the presence of the reset tone from $3.5 \%$ (see Fig. 2) to $0.7 \%$, demonstrating that the reset is in fact cooling the qubit below its effective bath occupation. (b) Cyclic operation of the pulse sequence in between the two vertical dashed lines, in the presence of a continuous input tone of variable amplitude (orange pulse). First, the qubit is reset for $2 \mu$ s (first green and purple pulses), followed by a heralding measurement to confirm the preparation in the ground state (second green pulse). The detector is then activated during a time $t_{p}=3 \mu$ s by switching on the pump (second purple pulse), and finally, the qubit state is read out (third green pulse). The measured $p_{e}$ (open circles) is plotted as a function of the photon number in the input pulse integrated over the cycle time $7 \mu \mathrm{s}$. A linear regression (solid line) yields an overall efficiency of $20 \%$ (which accounts for the $43 \%$ duty cycle) and a dark count of $2 \%$ per cycle. (c) Real-time trajectories of 1000 successive detection cycles, with $\bar{n}=0$ (top) and $\bar{n}=0.38$ (bottom), as marked by the full dot in (b).

readout [Fig. 3(a)]. The downtime of the detector comprises the reset, heralding, and readout pulses, and is as low as $3.5 \mu \mathrm{s}$ [Fig. 3(b)]. The cyclic operation is demonstrated by running the detector with a $43 \%$ duty cycle in the presence of a coherent tone on the buffer of variable strength [Fig. 3(c)]. Moreover, the qubit is at all times either in $|g\rangle$ or $|e\rangle$ (see Appendix E), and therefore a weak and continuous dispersive readout could be added to detect uninterruptedly the incoming photons with a precise timing of the clicks. Figures 3(c) and 3(d) are an example of 1000 samples from such measurement sequences in the absence of the probe tone giving a dark count rate of

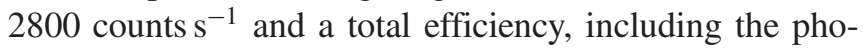
tons lost during the detector's downtime and $T_{1}$ event during the detection, given by $\eta_{\text {cyclic }}=0.19$. 
(a)

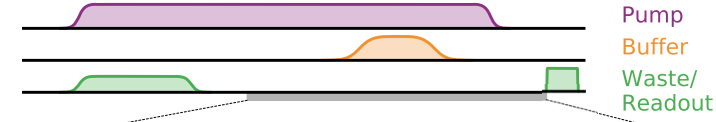

(b)

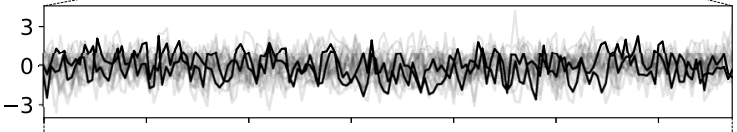

(c)
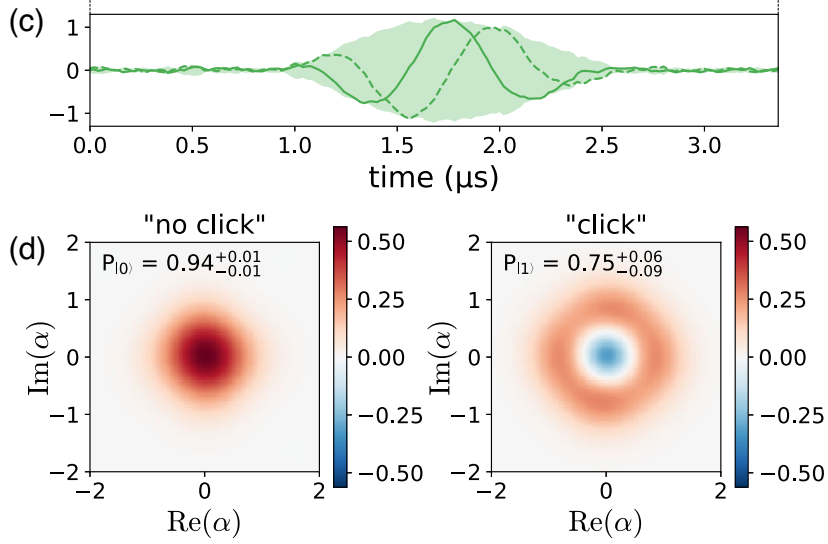

FIG. 4. Quantum nondemolition characterization by tomography of the quantum state released in the waste line. (a) The threewave-mixing interaction is activated by a pump tone (purple pulse) during which the detector is first initialized by a reset pulse on the waste port (first green pulse) preceding the injection of a small coherent field (mean occupation $\bar{n}=0.35$ ) on the buffer port. The qubit excitation, detected at the end of the sequence by a readout pulse (second green pulse), is correlated with the creation of a single photon by the waste. During the detection process, a near quantum-limited phase-preserving amplifier is used to monitor the field emitted by the waste (gray shaded rectangle). (b) The signal, demodulated at the frequency $\left(\omega_{w}^{g}+\omega_{w}^{e}\right) / 2$, is dominated by quantum and amplifier noise, as exemplified with 20 overlayed single-quadrature time traces ( 2 time traces have been colored in black for readability). (c) The waveform of the emitted wave packet can be deconvoluted from the uncorrelated detection noise by statistical analysis of the traces [38]. The real, imaginary part, and envelope of the waveform retrieved experimentally are represented in full line, dashed line, and green shaded area, respectively. (d) The quantum state of the field in the temporal mode plotted in (c) is reconstructed by analyzing the moments of the measured amplitude distribution [39] as described in Appendix $H$. The Wigner function of the state conditioned on the detection of the qubit in state $|g\rangle(|e\rangle)$ is represented on the left (right).

\section{QND-ness}

Finally, the waste which up to now has been considered as a bath can, in fact, be monitored to demonstrate that our detector is quantum nondemolition. Indeed, the qubit acts as a witness of the photon passage; the latter is first absorbed in the buffer and released through the waste at another carrier frequency [Fig. 1(b)]. By performing the heterodyne detection of the outgoing field, heralded by the click of the detector, we first extract the temporal mode of the photon based on the eigenmode expansion of the autocorrelation function [38]. Then, we reconstruct the density matrix of the photon by measuring the moments of the signal distribution up to the fourth order [39]. Based on a maximum likelihood reconstruction, we perform the tomography of the outgoing field (Fig. 4) and obtain a single photon probability $P_{|1\rangle}=$ $75_{-9}^{+6} \%$ conditioned on a click of the detector and a vacuum probability $P_{|0\rangle}=94_{-1}^{+1} \%$ conditioned on the absence of a click. The uncertainties were computed assuming a $\pm 0.5 \mathrm{~dB}$ calibration uncertainty on the gain of the amplification chain (see Appendix H). The source of infidelities can be carefully modeled based on the detector imperfections such as spurious thermal excitations, detection inefficiencies, readout infidelity, given the photon statistics of the itinerant coherent state. As described in Appendix H, we estimate the single photon probability $P_{|1\rangle}$ conditioned on a click of the detector to be $80 \%$ while the vacuum probability $P_{|0\rangle}$ conditioned on the absence of a click is estimated to be 94\%. These values are in agreement with the state reconstruction, thus validating the quantum nondemolition character of the detector.

\section{CONCLUSION}

We have realized a nonlinear and nonlocal dissipator involving a qubit coupled to a resonator. Conditioned on the impact of a photon on the resonator, the qubit irreversibly switches to its excited state which is then detected with a single-shot readout. This photon detector achieves both high efficiency and an unprecedentedly low dark count rate owing to its robustness against the main decoherence mechanisms of superconducting circuits. Following the example of Josephson parametric amplifiers in the advent of circuit quantum electrodynamics, this new class of robust detectors based on dissipation engineering constitutes an essential step toward the practical use of photon detectors in the microwave domain. By engineering higher-order dissipators, one can envision more complex detection patterns, such as number-resolving or multimode correlation detectors.

\section{ACKNOWLEDGMENTS}

Z.L. acknowledges support from the ANR grant ENDURANCE, and the EMERGENCES grant ENDURANCE of Ville de Paris. S. D. acknowledges support from the ANR grant QuNaT. E.I. acknowledges support from the European Union's Horizon 2020 Programme for Research and Innovation under Grant Agreement No. 722923 (Marie Curie ETN-OMT). E. A. acknowledges support from the European Union's Horizon 2020 research and innovation programme under the Marie Sklodowska-Curie Grant Agreement No. 765267. The devices were fabricated within the consortium Salle Blanche Paris Centre. E. F. acknowledges support from the ENS Junior Research Chair under grant LabEX ENSICFP: No. ANR-10-LABX-0010 and No. ANR-10-IDEX0001-02 PSL* and from the ANR grant DARKWADOR: ANR-19-CE47-0004. This work has been supported by the Paris Île-de-France Region in the framework of DIM SIRTEQ (DOMAINE D'INTÉRÊT MAJEUR SCIENCE ET INGÉNIERIE EN RÉGION ÎLE-DE-FRANCE POUR 
LES TECHNOLOGIES QUANTIQUES). E. F. and Z. L. conceived the experiment. R. L. and E.F. designed the circuit. R. L., U.R., and E.A. performed simulation of the chip device. R. L. fabricated the circuit. R. L., S. D., Z. L., and E. F. performed the experiment and analyzed the data. All authors contributed to the writing of the manuscript. All work was done under the direct supervision of S. D., Z. L., and E. F.

\section{APPENDIX A: CIRCUIT PARAMETERS}

The circuit consists of $\lambda / 2$-coplanar waveguide resonators as depicted in Fig. 5(b). The circuit is made out of sputtered niobium with a thickness of $120 \mathrm{~nm}$ deposited on a $280-\mu \mathrm{m}$-thick wafer of intrinsic silicon. The main circuit

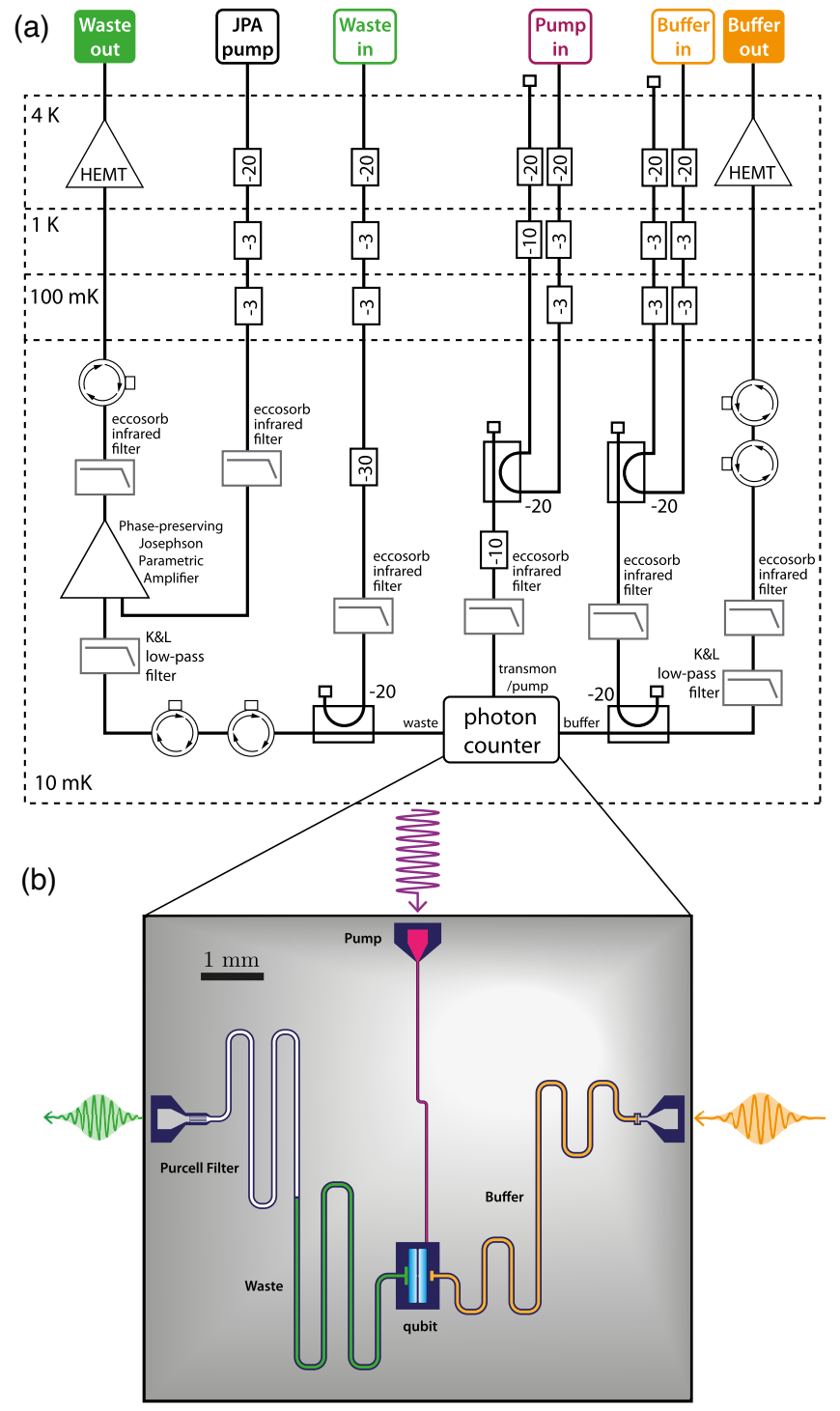

FIG. 5. (a) Schematic of microwave cabling of the dilution refrigerator. (b) Superconducting coplanar-waveguide layout; note that this vector layout is the exact mask used for the circuit fabrication.
TABLE I. Measured system parameters. Note that the internal losses of the waste are too small in comparison to the external losses to be accurately measured.

\begin{tabular}{lc}
\hline \hline Qubit & \\
\hline$\omega_{q} / 2 \pi$ & $4.532 \mathrm{GHz}$ \\
$T_{1}$ & $8-9 \mu \mathrm{s}$ \\
$T_{2}^{*}$ & $10 \mu \mathrm{s}$ \\
$\chi_{q q} / 2 \pi$ & $146 \mathrm{MHz}$ \\
$\chi_{q b} / 2 \pi$ & $1.02 \mathrm{MHz}$ \\
$\chi_{q w} / 2 \pi$ & $2.73 \mathrm{MHz}$ \\
\hline \hline
\end{tabular}

Waste mode

\begin{tabular}{lc}
\hline$\omega_{b}^{g} / 2 \pi$ & $5.7725 \mathrm{GHz}$ \\
$\kappa_{\text {ext }} / 2 \pi$ & $2.38 \mathrm{MHz}$ \\
$\kappa_{\text {int }} / 2 \pi$ & $<100 \mathrm{kHz}$
\end{tabular}

Buffer mode

$\omega_{w}^{g} / 2 \pi \quad 5.4952 \mathrm{GHz}$

$\kappa_{\text {ext }} / 2 \pi$

$0.890 \mathrm{MHz}$

$\kappa_{\text {int }} / 2 \pi$

$80 \mathrm{kHz}$

is etched after an optical lithography step and then the Josephson junction is made of evaporated aluminum through a PMMA/MAA resist mask written in a distinct $e$-beam lithography step.

\section{APPENDIX B: PURCELL FILTER}

In order to strongly couple the waste resonator to the cold $50 \Omega$ environment which provides the dissipative character of the scheme without degrading the qubit relaxation time $T_{1}$, we have employed a Purcell filter in series with the waste resonator as depicted in Fig. 5(b). As described in Ref. [40], another benefit of the Purcell filter is that the readout time of the qubit is reduced; hence, the readout fidelity is enhanced.

The Purcell filter is a bandpass filter consisting in a $\lambda / 2$ resonator coupled in series with the waste resonator at a rate $\mathcal{G}$ and placed on resonance with the latter, $\omega_{\text {Purcell }}=2 \pi \times 5.786 \mathrm{GHz}$, and coupled to the transmission line at a rate $\kappa_{\text {Purcell }}=2 \pi \times 36 \mathrm{MHz}$. The filter is designed to be in the strong damping regime $\kappa_{\text {Purcell }} \gg \mathcal{G}$ so that the expected anticrossing due to the hybridization of the modes is irrelevant. The effective decay rate of the waste resonator to the transmission line through the filter is then given by

$$
\begin{aligned}
\kappa_{w} & =\frac{4 \mathcal{G}^{2}}{\kappa_{\text {Purcell }}} \frac{1}{1+\left[2\left(\omega_{\text {Purcell }}-\omega_{w}\right) / \kappa_{\text {Purcell }}\right]^{2}} \\
& \approx \frac{4 \mathcal{G}^{2}}{\kappa_{\text {Purcell }}} .
\end{aligned}
$$


Experimentally, we extract an effective decay rate of $\kappa_{w}=2 \pi \times 2.38 \mathrm{MHz}$ leading to a coupling strength $\mathcal{G}=2 \pi \times 5.6 \mathrm{MHz}$ between the waste and the Purcell filter. The qubit is coupled to the waste resonator at a rate $g=2 \pi \times 41 \mathrm{MHz}$. Its residual decay rate through the waste channel in the presence of the Purcell filter is given by

$$
\begin{aligned}
\kappa_{q}^{(w)} & =\frac{g^{2}}{\left(\omega_{w}-\omega_{q}\right)^{2}} \frac{4 \mathcal{G}^{2}}{\kappa_{\text {Purcell }}} \frac{1}{1+\left[2\left(\omega_{\text {Purcell }}-\omega_{q}\right) / \kappa_{\text {Purcell }}\right]^{2}} \\
& \approx \frac{g^{2} \mathcal{G}^{2}}{\left(\omega_{w}-\omega_{q}\right)^{4}} \kappa_{\text {Purcell }}=2 \pi \times 1 \mathrm{~Hz} .
\end{aligned}
$$

Note that for a given decay rate of the waste in the transmission line, the Purcell filter enables the suppression of the qubit relaxation through the waste channel by a factor $\kappa_{\text {Purcell }}^{2} /\left(\omega_{\text {Purcell }}-\omega_{q}\right)^{2} \sim 10^{-3}$.

\section{APPENDIX C: SYSTEM HAMILTONIAN DERIVATION}

Our system consists of three electromagnetic modes, referred to as the buffer, the waste, and the qubit, coupled through a Josephson junction. A strong radio-frequency drive, referred to as the pump, is applied to the qubit mode. The Hamiltonian of this system is well described by

$$
\begin{aligned}
\hat{H} / \hbar= & \sum_{m=b, w, q} \omega_{m} \hat{m}^{\dagger} \hat{m} \\
& -\frac{E_{J}}{\hbar}\left[\cos (\hat{\varphi})+\hat{\varphi}^{2} / 2\right]+2 \epsilon_{p} \cos \left(\omega_{p} t\right)\left(\hat{q}+\hat{q}^{\dagger}\right), \\
\hat{\varphi}= & \sum_{m=b, w, q} \varphi_{m}\left(\hat{m}+\hat{m}^{\dagger}\right),
\end{aligned}
$$

where the index $m=b, w, q$ refers to the buffer, waste, and qubit modes, respectively, of angular frequency $\omega_{m}$, and annihilation operator $\hat{m}$. The Josephson energy is denoted $E_{J}$, and $\hat{\varphi}$ is the phase across the junction, which can be decomposed as the sum of the phase $\varphi_{m}\left(\hat{m}+\hat{m}^{\dagger}\right)$ across each mode, where $\varphi_{m}$ is the zero point fluctuation of the phase across mode $m$. The pump's amplitude and angular frequency are denoted $\epsilon_{p}$ and $\omega_{p}$, respectively.

The strong pump is accounted for by moving into a frame where the qubit mode is displaced by its mean field amplitude $\xi_{p} e^{-i \omega_{p} t}$, where $\xi_{p} \approx-\epsilon_{p} /\left(\omega_{q}-\omega_{p}\right)$ [30], provided that the pump is adiabatically switched on and off with respect to the detuning $\omega_{q}-\omega_{p}$ (about $300 \mathrm{MHz}$ in our experiment) [41].

Following this frame displacement, we place ourselves in the interaction picture with respect to the Hamiltonian $\sum_{m=b, w, q}\left(\omega_{m}-\delta_{m}\right) \hat{m}^{\dagger} \hat{m}$, where $\delta_{m}$ are arbitrary detunings that will be used to cancel the ac-Stark shifts due to the Kerr effect. The transformed Hamiltonian reads:

$$
\begin{aligned}
\hat{H}^{\prime} / \hbar= & \sum_{m=b, w, q} \delta_{m} \hat{m}^{\dagger} \hat{m}-\frac{E_{J}}{\hbar}\left[\cos \left(\hat{\varphi}^{\prime}\right)+\hat{\varphi}^{\prime 2} / 2\right], \\
\hat{\varphi}^{\prime}= & \sum_{m=b, w, q} \varphi_{m}\left(\hat{m} e^{-i\left(\omega_{m}-\delta_{m}\right) t}+\hat{m}^{\dagger} e^{i\left(\omega_{m}-\delta_{m}\right) t}\right) \\
& +\varphi_{q}\left(\xi_{p} e^{-i \omega_{p} t}+\xi_{p}^{*} e^{i \omega_{p} t}\right) .
\end{aligned}
$$

We now take

$$
\omega_{p}=\left(\omega_{q}-\delta_{q}\right)+\left(\omega_{w}-\delta_{w}\right)-\left(\omega_{b}-\delta_{b}\right),
$$

and expanding the cosine term to fourth order, and keeping only the nonrotating terms leads to

$$
\begin{aligned}
\hat{H}^{\prime} & \approx H_{\text {Stark }}+H_{\mathrm{Kerr}}+H_{4 W M}, \\
\hat{H}_{\text {Stark }} / \hbar & =\sum_{m=b, w}\left(\delta_{m}-\chi_{q m}\left|\xi_{p}\right|^{2}\right) \hat{m}^{\dagger} \hat{m}+\left(\delta_{q}-2 \chi_{q q}\left|\xi_{p}\right|^{2}\right) \hat{q}^{\dagger} \hat{q}, \\
\hat{H}_{\mathrm{Kerr}} / \hbar & =\sum_{m=b, w, q}-\frac{\chi_{m m}}{2} \hat{m}^{\dagger 2} \hat{m}^{2}-\chi_{q b} \hat{b} \hat{b}^{\dagger} \hat{b} \hat{q}^{\dagger} \hat{q} \\
& -\chi_{q w} \hat{w}^{\dagger} \hat{w} \hat{q}^{\dagger} \hat{q}-\chi_{b w} \hat{b}^{\dagger} \hat{b} \hat{w}^{\dagger} \hat{w}, \\
\hat{H}_{4 W M} / \hbar & =g_{3} \hat{b} \hat{w}^{\dagger} \hat{q}^{\dagger}+g_{3}^{*} \hat{b}^{\dagger} \hat{w} \hat{q},
\end{aligned}
$$

with the coefficients $\hbar \chi_{m m}=E_{J} \phi_{m}^{4} / 2, \hbar \chi_{q b}=E_{J} \phi_{q}^{2} \phi_{b}^{2}$, $\hbar \chi_{q w}=E_{J} \phi_{q}^{2} \phi_{w}^{2}, \hbar \chi_{b w}=E_{J} \phi_{b}^{2} \phi_{w}^{2}$, and $\hbar g_{3}=-E_{J} \xi_{p} \phi_{q}^{2} \phi_{b} \phi_{w}$, which can also be written as

$$
g_{3}=-\xi_{p} \sqrt{\chi_{q b} \chi_{q w}}
$$

Note that we have neglected terms of the form $\hat{m}^{\dagger} \hat{m}$ arising from the normal ordering of the fourth-order term, since they simply shift the bare frequencies $\omega_{m}$ by a constant amount. Since the qubit anharmonicity $\chi_{q q}$ is much larger than all the dissipation and excitation rates, in the following, we project the qubit mode onto its two lowest energy levels $|g\rangle$ and $|e\rangle$. We thus replace the bosonic operator $\hat{q}$ by the two-level lowering operator $\hat{\sigma}=|g\rangle\langle e|$. Moreover, we choose the mode reference frames such that $\delta_{q}=2 \chi_{q q}\left|\xi_{p}\right|^{2}, \quad \delta_{b}=\chi_{q b}\left|\xi_{p}\right|^{2}$, and $\delta_{w}=\chi_{q w}\left|\xi_{p}\right|^{2}+\Delta$. We have introduced an arbitrary detuning $\Delta$ which, as we see in the next section, can be chosen to cancel the effect of the cross-Kerr effect between the qubit and the waste $\chi_{q w}$. This leads to $\hat{H}_{\text {Stark }} / \hbar=\Delta \hat{w}^{\dagger} \hat{w}$. The pump frequency thus needs to be adapted for each value of $\xi_{p}$ in order to always verify Eq. (C2):

$\omega_{p}=\omega_{q}+\omega_{w}-\omega_{b}-\Delta-\left|\xi_{p}\right|^{2}\left(2 \chi_{q q}+\chi_{q w}-\chi_{q b}\right)$. 
The Hamiltonian now reads:

$$
\begin{aligned}
\hat{H}^{\prime \prime} / \hbar= & g_{3} \hat{b} \hat{w}^{\dagger} \hat{\sigma}^{\dagger}+g_{3}^{*} \hat{b}^{\dagger} \hat{w} \hat{\sigma}+\Delta \hat{w}^{\dagger} \hat{w} \\
& +\sum_{m=b, w}-\frac{\chi_{m m}}{2} \hat{m}^{\dagger 2} \hat{m}^{2}-\chi_{q b} \hat{b}^{\dagger} \hat{b} \hat{\sigma}^{\dagger} \hat{\sigma} \\
& -\chi_{q w} \hat{w}^{\dagger} \hat{w} \hat{\sigma}^{\dagger} \hat{\sigma}-\chi_{b w} \hat{b}^{\dagger} \hat{b} \hat{w}^{\dagger} \hat{w} .
\end{aligned}
$$

\section{APPENDIX D: ADIABATIC ELIMINATION OF THE WASTE MODE}

A crucial resource in our system is the intentional dissipation of the waste mode $w$, which is coupled to a transmission line with a rate $\kappa_{w}$. In addition, the buffer mode is overcoupled (rate $\kappa_{w}$ ) to the input line which carries the incoming photons. Finally, the qubit mode is designed to be isolated from its environment, but inevitably has a residual uncontrolled dissipation at a rate $\kappa_{q}$, and dephasing $\kappa_{\phi}$.

$$
\begin{aligned}
\frac{d}{d t} \rho= & -i\left[\hat{H}^{\prime \prime} / \hbar, \rho\right]+\kappa_{w} \mathcal{D}[\hat{w}] \rho+\kappa_{b} \mathcal{D}[\hat{b}] \rho \\
& +\kappa_{q} \mathcal{D}[\hat{\sigma}] \rho+\frac{\kappa_{\phi}}{2} \mathcal{D}\left[\hat{\sigma}_{z}\right] \rho
\end{aligned}
$$

where the Lindblad operator is defined for any operator $\hat{O}$ as $\mathcal{D}[\hat{O}] \rho=\hat{O} \rho \hat{O}^{\dagger}-\frac{1}{2} \hat{O}^{\dagger} \hat{O} \rho-\frac{1}{2} \rho \hat{O}^{\dagger} \hat{O}$.

We place ourselves in the regime where $\left|g_{3}\right|, \chi_{q b}, \chi_{b w}$, $\chi_{b b}, \kappa_{b}, \kappa_{q}, \kappa_{\phi} \sim \delta \kappa_{w}$, and $\delta$ is a small parameter $\delta \ll 1$. In our experiment, $\chi_{q w} / \kappa_{w} \sim 1$, and we assume $\Delta / \kappa_{w} \sim 1$. In this regime, the waste mode can be adiabatically eliminated, leading to an effective dynamics for the buffer and qubit modes alone. Following Ref. [30], we search for a solution of the full buffer-qubit-waste dynamics of the form

$$
\begin{aligned}
\rho= & \rho_{00} \otimes|0\rangle\langle 0|+\delta\left(\rho_{10} \otimes|1\rangle\left\langle 0\left|+\rho_{01} \otimes\right| 0\right\rangle\langle 1|\right) \\
& +\delta^{2}\left(\rho_{11} \otimes|1\rangle\left\langle 1\left|+\rho_{20} \otimes\right| 2\right\rangle\left\langle 0\left|+\rho_{02} \otimes\right| 0\right\rangle\langle 2|\right) \\
& +O\left(\delta^{3}\right),
\end{aligned}
$$

where $\rho_{m n}=\langle m|\rho| n\rangle$ is the reduced density matrix acting on the buffer Hilbert space with $|m\rangle,|n\rangle$ being the Fock states basis of the waste mode. Whereas $|n\rangle\langle m|$ acts on the waste Hilbert space. The goal here is to derive the dynamics of $\rho_{q b}=\operatorname{Tr}_{w}(\rho)=\rho_{00}+\delta^{2} \rho_{11}$ up to second order in $\delta$, where $\operatorname{Tr}_{w}$ denotes the partial trace over the waste degrees of freedom. The low occupancy of the waste mode justifies this expansion. We are interested in the dynamics of the reduced density operator for the qubit-buffer modes $\rho_{q b}$, which is obtained by taking the partial trace over the waste mode. We rewrite the Hamiltonian of Eq. (D6) in the following form,

$$
\begin{aligned}
\hat{H}^{\prime \prime} / \hbar= & g_{3} \hat{b} \hat{\sigma}^{\dagger} \hat{w}^{\dagger}+g_{3}^{*} \hat{b}^{\dagger} \hat{\sigma} \hat{w} \\
& +\left(\Delta-\chi_{q w} \hat{\sigma}^{\dagger} \hat{\sigma}-\chi_{b w} \hat{b}^{\dagger} \hat{b}\right) \hat{w}^{\dagger} \hat{w}+\hat{H}_{q b} / \hbar, \\
\hat{H}_{q b} / \hbar= & -\frac{\chi_{b b}}{2} \hat{b}^{\dagger 2} \hat{b}^{2}-\chi_{q b} \hat{b}^{\dagger} \hat{b} \hat{\sigma}^{\dagger} \hat{\sigma},
\end{aligned}
$$

and we define

$$
\begin{aligned}
\mathcal{L}_{q b}\left(\rho_{q b}\right)= & -\frac{i}{\hbar}\left[\hat{H}_{q b}, \rho_{q b}\right] \\
& +\kappa_{b} \mathcal{D}[\hat{b}] \rho_{q b}+\kappa_{q} \mathcal{D}[\hat{\sigma}] \rho_{q b}+\frac{\kappa_{\phi}}{2} \mathcal{D}\left[\hat{\sigma}_{z}\right] \rho_{q b} .
\end{aligned}
$$

By projecting Eq. (D1) with $\langle 0|\ldots| 0\rangle,\langle 0|\ldots| 1\rangle$, and $\langle 1|\ldots| 1\rangle$, respectively, we get

$\frac{1}{\kappa_{w}} \frac{d}{d t} \rho_{00}=\delta^{2}\left(i \rho_{01} \hat{A}-i \hat{A}^{\dagger} \rho_{10}+\rho_{11}\right)+\frac{1}{\kappa_{w}} \mathcal{L}_{q b}\left(\rho_{00}\right)+O\left(\delta^{3}\right)$,

$$
\begin{aligned}
& \frac{1}{\kappa_{w}} \frac{d}{d t} \rho_{01}=i \rho_{00} \hat{A}^{\dagger}-\rho_{01}\left(\frac{1}{2}-i \hat{\Delta}\right)+O(\delta), \\
& \frac{1}{\kappa_{w}} \frac{d}{d t} \rho_{11}=i \rho_{10} \hat{A}^{\dagger}-i \hat{A} \rho_{01}-i\left[\hat{\Delta}, \rho_{11}\right]-\rho_{11}+O(\delta),
\end{aligned}
$$

where

$$
\begin{aligned}
& \hat{A}=\frac{g_{3}}{\kappa_{w} \delta} \hat{b} \hat{\sigma}^{\dagger}, \\
& \hat{\Delta}=\frac{\Delta-\chi_{q w} \hat{\sigma}^{\dagger} \hat{\sigma}}{\kappa_{w}} .
\end{aligned}
$$

Note that $\|\hat{A}\|$ and $\|\hat{\Delta}\|$ are of order $\delta^{0}$. Considering Eq. (D6), we see that the derivative of $\rho_{01}$ is composed of a term proportional to $\rho_{00}$, that can be viewed as an external drive, and a term proportional to $\rho_{01}$, that includes a damping term. Since the variation of $\rho_{00}$ is slow $\left[d \rho_{00} / \kappa_{w} d t\right.$ of order $\delta^{2}$; see Eq. (C5)] in comparison to the damping term (of order $\delta^{0}$ ), we can make the adiabatic approximation: we consider that $\rho_{01}$ is continuously in its steady state. The same reasoning applies to $\rho_{11}$; we thus set to 0 the derivatives of the left-hand side of Eqs. (D6) and (D7). Moreover, by noting that $\hat{\Delta} \hat{A}=$ $\left(\Delta-\chi_{q w}\right) \hat{A} / \kappa_{w}$, we can solve for $\rho_{01}, \rho_{10}, \rho_{11}$ as a function of $\rho_{00}$. We find

$$
\begin{aligned}
& \rho_{01}=\frac{1}{1+4\left|\frac{\Delta-\chi_{q w}}{\kappa_{w}}\right|^{2}}\left(2 i-4 \frac{\left(\Delta-\chi_{q w}\right)}{\kappa_{w}}\right) \rho_{00} \hat{A}^{\dagger}, \\
& \rho_{11}=\frac{1}{1+4\left|\frac{\Delta-\chi_{q w}}{\kappa_{w}}\right|^{2}} 4 \hat{A} \rho_{00} \hat{A}^{\dagger} .
\end{aligned}
$$


We denote

$$
\begin{aligned}
\kappa_{\mathrm{nl}} & =\frac{4\left|g_{3}\right|^{2} / \kappa_{w}}{1+4\left|\frac{\Delta-\chi_{q w}}{\kappa_{w}}\right|^{2}}, \\
\Delta_{\mathrm{nl}} & =\frac{4\left|g_{3}\right|^{2} / \kappa_{w}}{1+4\left|\frac{\Delta-\chi_{q w}}{\kappa_{w}}\right|^{2}} \frac{\chi_{q w}-\Delta}{\kappa_{w}} .
\end{aligned}
$$

Inserting the solutions (D10) and (D11) into Eq. (D5), we find

$$
\begin{aligned}
\frac{d}{d t} \rho_{00}= & -i \Delta_{\mathrm{nl}}\left[\hat{b}^{\dagger} \hat{b} \hat{\sigma} \sigma^{\dagger}, \rho_{00}\right] \\
& +\kappa_{\mathrm{nl}} \mathcal{D}\left[\hat{b} \hat{\sigma}^{\dagger}\right] \rho_{00}+\mathcal{L}_{q b}\left(\rho_{00}\right)+O\left(\delta^{3}\right) .
\end{aligned}
$$

The term proportional to $\kappa_{\mathrm{nl}}$ is the nonlinear damping term at the heart of the single microwave photon detector. It is maximized for $\Delta=\chi_{q w}$. In this configuration, the pump angular frequency $\omega_{p}=\omega_{q}+\omega_{w}-\omega_{b}-\chi_{q w}$ is such that $\hbar \omega_{p}$ exactly matches the energy difference between the initial state $\hat{b}^{\dagger}|0,0\rangle|0\rangle=|1,0\rangle|0\rangle\left(\omega_{b}\right)$ and final state $\hat{\sigma}^{\dagger} \hat{w}^{\dagger}|0,0\rangle|0\rangle=|0,1\rangle|1\rangle\left(\omega_{q}+\omega_{w}-\chi_{q w}\right)$, and $\kappa_{\mathrm{nl}}=4\left|g_{3}\right|^{2} / \kappa_{w}$. The term proportional to $\Delta_{\mathrm{nl}}$ is a "generalized frequency pull" that corresponds to a tunable crossKerr effect between the qubit and buffer modes. Note that $\rho_{q b}=\rho_{00}+\delta^{2} \rho_{11}$ follows the same dynamics as Eq. (D14) (since we assumed $d \rho_{11} / \kappa_{w} d t=0$ ).

\section{APPENDIX E: QUBIT DYNAMICS AND DETECTION EFFICIENCY}

\section{Efficiency for single photon Fock states}

The detector efficiency is defined as the probability $p_{e}$ to find the qubit in the excited state, when a Fock state $|1\rangle$ is incident on the buffer cavity. However, in this work, we calibrate our photon detector with coherent states. In the following, we provide a simple argument to bridge the gap between this definition of $\eta$ and the experiment performed with coherent states.

We can formally describe the output of a random source that emits a single photon with a small probability $\epsilon$ with the density matrix

$$
\rho=(1-\varepsilon)|0\rangle\langle 0|+\varepsilon| 1\rangle\langle 1| .
$$

With such a source, the detector should click with a probability $p_{e}=\eta \epsilon$. Additionally, a coherent state with a small complex amplitude $\alpha$ reads

$$
|\psi\rangle \approx\left(1-\frac{1}{2}|\alpha|^{2}\right)(|0\rangle+\alpha|1\rangle),
$$

and thus the statistical mixture of coherent states with unknown phase reads

$$
\rho \approx\left(1-|\alpha|^{2}\right)|0\rangle\left\langle\left. 0|+| \alpha\right|^{2} \mid 1\right\rangle\langle 1| .
$$

Therefore, one can identify this statistical mixture with an intermittent single photon source providing that $\varepsilon=|\alpha|^{2}$ is small. The probability of click will then be $p_{e}=\eta \varepsilon=\eta \bar{n}$ in either case.

\section{Efficiency for coherent states}

In order to describe the effect of coherent states on the detector and to compute $\eta$, we now add an input drive of amplitude $\epsilon=\sqrt{\kappa_{b}} b_{\text {in }}$, where $b_{\text {in }}$ is the amplitude of the coherent pulse. We start from Eq. (D14), where we neglect $\chi_{b b}, \chi_{q b}, \kappa_{q} \ll \kappa_{\mathrm{nl}}, \kappa_{b}$, and take $\Delta=\chi_{q w}$ :

$\frac{d}{d t} \rho_{q b}=\kappa_{\mathrm{nl}} \mathcal{D}\left[\hat{b} \hat{\sigma}^{\dagger}\right] \rho_{q b}+\kappa_{b} \mathcal{D}[\hat{b}] \rho_{q b}+\epsilon\left[\hat{b}-\hat{b}^{\dagger}, \rho_{q b}\right]$.

We would now like to calculate the qubit excited state population $p_{e}$ as a function of the number of photons in the incoming wave packet of length $T: \bar{n}_{\text {in }}=\left|b_{\text {in }}\right|^{2} \times T$, where we assume for simplicity that $b_{\text {in }}$ is time independent. We may write the solution of Eq. (E4) in the general form $\rho_{q b}=\rho_{g g}|g\rangle\left\langle g\left|+\rho_{g e}\right| g\right\rangle\left\langle e\left|+\rho_{e g}\right| e\right\rangle\left\langle g\left|+\rho_{e e}\right| e\right\rangle\langle e|$, and we are interested in $p_{e}=\operatorname{Tr}\left(\rho_{e e}\right)$. We find

$$
\begin{aligned}
\frac{d}{d t} \rho_{g g}= & -\frac{\kappa_{\mathrm{nl}}}{2}\left(\hat{b}^{\dagger} \hat{b} \rho_{g g}+\rho_{g g} \hat{b}^{\dagger} \hat{b}\right) \\
& +\kappa_{b} \mathcal{D}[\hat{b}] \rho_{g g}+\epsilon\left[\hat{b}-\hat{b}^{\dagger}, \rho_{g g}\right], \\
\frac{d}{d t} \rho_{e e}= & \kappa_{\mathrm{nl}} \hat{b} \rho_{g g} \hat{b}^{\dagger}+\kappa_{b} \mathcal{D}[\hat{b}] \rho_{e e}+\epsilon\left[\hat{b}-\hat{b}^{\dagger}, \rho_{e e}\right] .
\end{aligned}
$$

Hence,

$$
\frac{d}{d t} p_{e}=\kappa_{\mathrm{nl}} \operatorname{Tr}\left(\hat{b} \rho_{g g} \hat{b}^{\dagger}\right),
$$

and we now need to solve Eq. (C5) for which, remarkably, we find a simple ansatz:

$$
\begin{aligned}
\rho_{g g}(t) & =\exp \left(-4 \frac{\epsilon^{2}}{\left(\kappa_{\mathrm{nl}}+\kappa_{b}\right)^{2}} \kappa_{\mathrm{nl}} t\right)|\beta\rangle\langle\beta|, \\
\beta & =-2 \epsilon /\left(\kappa_{\mathrm{nl}}+\kappa_{b}\right),
\end{aligned}
$$

where $|\beta\rangle$ is a coherent state of amplitude $\beta$.

Note that $\operatorname{Tr}\left(\hat{b} \rho_{g g} \hat{b}^{\dagger}\right)=\beta^{2} \exp \left\{-4\left[\epsilon^{2} /\left(\kappa_{\mathrm{nl}}+\kappa_{b}\right)^{2}\right] \kappa_{\mathrm{nl}} t\right\}$, and hence inserting this expression in Eq. (E7), we find

$\frac{d}{d t} p_{e}=4 \kappa_{\mathrm{nl}} \frac{\epsilon^{2}}{\left(\kappa_{\mathrm{nl}}+\kappa_{b}\right)^{2}} \exp \left(-4 \frac{\epsilon^{2}}{\left(\kappa_{\mathrm{nl}}+\kappa_{b}\right)^{2}} \kappa_{\mathrm{nl}} t\right)$.

Assuming the qubit in its ground state at $t=0$, we find

$$
p_{e}(T)=1-\exp \left(-\eta\left|b_{\text {in }}\right|^{2} T\right),
$$


where

$$
\eta=4 \frac{\kappa_{b} \kappa_{\mathrm{nl}}}{\left(\kappa_{\mathrm{nl}}+\kappa_{b}\right)^{2}}
$$

which can be written as

$$
p_{e}=1-\mathbb{P}_{0}^{\eta} \underset{\bar{n}_{\text {in }} \rightarrow 0}{\sim} \eta \bar{n}_{\text {in }} .
$$

Here $\mathbb{P}_{0}=\exp \left(-\bar{n}_{\text {in }}\right)$ is the population of Fock state 0 of the incoming coherent pulse, which has a mean photon number $\bar{n}_{\text {in }}=\left|b_{\text {in }}\right|^{2} \times T$ and follows a Poisson distribution. We see from Eq. (4) that $\eta=1$ when $\kappa_{\mathrm{nl}}=\kappa_{b}$.

\section{Time-dependent pulses}

This derivation was made for a time-independent pulse amplitude $\epsilon$, but one arrives to a similar result for $\epsilon(t)$. We start from Eqs. (C5) and (E6), where $\epsilon$ is replaced by a time-dependent pulse $\epsilon(t)$. We assume that $\epsilon(t)$ represents a wave packet so that $\epsilon(t \rightarrow-\infty)=\epsilon(t \rightarrow \infty)=0$. We define $\tilde{\rho}_{g g}$ such as

$$
\frac{d}{d t} \tilde{\rho}_{g g}=\left(\kappa_{b}+\kappa_{\mathrm{nl}}\right) \mathcal{D}[\hat{b}] \tilde{\rho}_{g g}+\epsilon(t)\left[\hat{b}-\hat{b}^{\dagger}, \tilde{\rho}_{g g}\right]
$$

which is the intracavity state of a two-port driven cavity. Importantly, this is a coherent state at all time. We can show that

$$
\rho_{g g}(t)=f(t) \tilde{\rho}_{g g}(t)
$$

is the solution of Eq. (E5) with a time-dependent pulse, where

$$
\begin{aligned}
f(t) & =\exp \left(-\kappa_{\mathrm{nl}} \int_{0}^{t}|\beta(t)|^{2} d t\right), \\
|\beta(t)|^{2} & =\operatorname{Tr}\left(\hat{b} \tilde{\rho}_{g g}(t) \hat{b}^{\dagger}\right),
\end{aligned}
$$

and $\beta(t)$ is easy to compute. We have that

$$
\frac{d}{d t} p_{e}=\kappa_{\mathrm{nl}} \operatorname{Tr}\left(\hat{b} \rho_{g g} \hat{b}^{\dagger}\right)=\kappa_{\mathrm{nl}} f(t)|\beta(t)|^{2},
$$

so we can find the occupation of the qubit after the pulse $p_{e}(+\infty) \equiv p_{e}=1-f(+\infty)$ :

$$
\begin{aligned}
p_{e} & =1-\exp \left(-\kappa_{\mathrm{nl}} \int_{-\infty}^{+\infty}|\beta(t)|^{2} d t\right) \\
& =1-\exp \left(-\kappa_{\mathrm{nl}} \frac{1}{2 \pi} \int_{-\infty}^{+\infty}|\beta(\omega)|^{2} d \omega\right) \\
& =1-\exp \left(-\frac{1}{2 \pi} \int_{-\infty}^{+\infty} \kappa_{b} \kappa_{\mathrm{nl}}\left|\frac{b_{\mathrm{in}}(\omega)}{\left(\kappa_{\mathrm{nl}}+\kappa_{b}\right) / 2+i \omega}\right|^{2} d \omega\right),
\end{aligned}
$$

where $b_{\text {in }}(\omega)$ is the incoming pulse in the frequency domain. We can rewrite this as

$$
p_{e}=1-\exp \left(-\bar{n}_{\text {cav }}\right)=1-\mathbb{P}_{0, \text { cav }},
$$

where $\bar{n}_{\text {cav }}$ is the overall number of photons that actually reach into the buffer. This is to be compared to

$$
\bar{n}_{\text {pulse }}=\frac{1}{2 \pi} \int_{-\infty}^{+\infty}\left|b_{\text {in }}(\omega)\right|^{2} d \omega,
$$

the number of photons in the incoming pulse. Equation (E19) shows the filtering of the incoming pulse due to the loss mismatch $\left(\kappa_{b} \neq \kappa_{\mathrm{nl}}\right)$ of the detector and its finite bandwidth $\left(\kappa_{b}+\kappa_{\mathrm{nl}}\right)$.

\section{APPENDIX F: RESET PROTOCOL}

For the reset procedure, we switch off the drive on the buffer mode, and instead, we add a resonant drive on the waste port (this drive is sent at $\omega_{w}-\chi_{q w}$ since the qubit is in $|e\rangle$ when we want to reset). This adds to the Hamiltonian of Eq. (D3) the following term, $H_{\text {drive }}=\epsilon_{w}\left(\hat{w}+\hat{w}^{\dagger}\right)$, which can be absorbed by replacing the operator $\hat{b} \hat{\sigma}^{\dagger} \rightarrow$ $\hat{b} \hat{\sigma}^{\dagger}+\epsilon_{w} / g_{3}$. Using this modified expression, the rest of the calculation follows. Note that

$\kappa_{\mathrm{nl}} \mathcal{D}\left[\hat{b} \hat{\sigma}^{\dagger}+\frac{\epsilon_{w}}{g_{3}}\right] \rho_{q b}=\kappa_{\mathrm{nl}} \mathcal{D}\left[\hat{b} \hat{\sigma}^{\dagger}\right] \rho_{q b}+\kappa_{\mathrm{nl}} \frac{\epsilon_{w}}{2 g_{3}}\left[\hat{b} \hat{\sigma}^{\dagger}-\hat{b}^{\dagger} \hat{\sigma}, \rho_{q b}\right]$.

This leads to

$$
\begin{aligned}
\frac{d}{d t} \rho_{q b}= & \kappa_{b} \mathcal{D}[\hat{b}] \rho_{q b}+\kappa_{\mathrm{nl}} \mathcal{D}\left[\hat{b} \hat{\sigma}^{\dagger}\right] \rho_{q b} \\
& +\epsilon_{\mathrm{nl}}\left[\hat{b} \hat{\sigma}^{\dagger}-\hat{b}^{\dagger} \hat{\sigma}, \rho_{q b}\right]
\end{aligned}
$$

where $\epsilon_{\mathrm{nl}}=\kappa_{\mathrm{nl}}\left(\epsilon_{w} / 2 g_{3}\right)$. Instead of repeating the entire analysis of the previous sections, we will proceed using a useful analogy. We see that Eq. (F2) can be mapped to Eq. (E4) by making the following substitutions: $\kappa_{b} \leftrightarrow \kappa_{\mathrm{nl}}$, $\hat{b} \leftrightarrow \hat{b} \hat{\sigma}^{\dagger}$, and $\epsilon_{\mathrm{nl}} \leftrightarrow \epsilon$. We have shown that the dynamics of Eq. (E4) leads the qubit to dissipate from its ground to its excited state at rate $4\left[\epsilon^{2} /\left(\kappa_{\mathrm{nl}}+\kappa_{b}\right)^{2}\right] \kappa_{\mathrm{nl}}$ [see Eq. (E11)]. By 
analogy, the dynamics of Eq. (F2) leads the qubit to dissipate from its excited state to its ground state at a rate

$$
\begin{aligned}
\kappa_{\text {reset }} & =4 \frac{\epsilon_{\mathrm{nl}}^{2}}{\left(\kappa_{b}+\kappa_{\mathrm{nl}}\right)^{2}} \kappa_{b} \\
& =4 \frac{\epsilon_{w}^{2}}{\left(\kappa_{b}+\kappa_{\mathrm{nl}}\right)^{2}} \kappa_{\mathrm{nl}} \frac{\kappa_{b}}{\kappa_{w}} .
\end{aligned}
$$

\section{APPENDIX G: SPECTROSCOPIC CHARACTERIZATION OF THE DETECTOR}

When inserting the optimal pump frequency predicted by Eq. (D14) $\left(\Delta=\chi_{q w}\right)$ into the frequency matching condition (C5), we find

$$
\begin{aligned}
\omega_{p}= & \left(\omega_{q}-2 \chi_{q q}\left|\xi_{p}\right|^{2}\right)+\left[\omega_{w}-\chi_{q w}\left(1+\left|\xi_{p}\right|^{2}\right)\right] \\
& -\left(\omega_{b}-\chi_{q b}\left|\xi_{p}\right|^{2}\right) .
\end{aligned}
$$

This equation is equivalent to Eq. (1) with $\bar{\omega}_{q} \equiv$ $\omega_{q}-2 \chi_{q q}\left|\xi_{p}\right|^{2}, \quad \omega_{w}^{e} \equiv \omega_{w}-\chi_{q w}\left(1+\left|\xi_{p}\right|^{2}\right), \quad$ and $\quad \omega_{b}^{g} \equiv$ $\omega_{g}-\chi_{q b}\left|\xi_{p}\right|^{2}$. Note that in the main text, for clarity, we have neglected the ac-Stark shifts on the buffer and waste since $\left|\xi_{p}\right|^{2} \chi_{q b, w}<\left|g_{3}\right|$. To match this condition, we realize

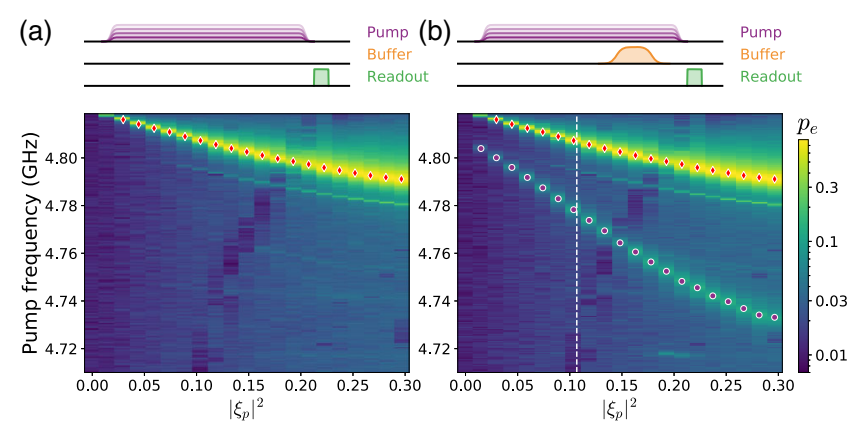

FIG. 6. Complete spectroscopic data. (a),(b) The excited state population of the qubit $p_{e}$ (color coded) is represented as a function of the pump amplitude ( $x$ axis) and pump frequency ( $y$ axis). (a) When there is no incoming photon on the buffer port, we find one spectroscopic line (red diamonds) for which the qubit is sent into its excited state. This corresponds to a higher-order amplification process of the form. We also observe that the background $p_{e}$ is rising approximately linearly with the pump power at all the frequencies, which is consistent with the heating of the qubit bath by the pump. (b) When we add a pulse on the buffer port at frequency $\omega_{b}$, another line (purple circles) appears. This line corresponds the process $\hat{b} \hat{\sigma}^{\dagger} \hat{w}^{\dagger}$ and the white dashed line indicates the chosen pump power which maximize the ratio $\eta / \Gamma_{\mathrm{dc}}$. We can also observe a spectroscopic line which goes upward which seems to cool the qubit and thus decreases the efficiency [see dip in $\kappa_{\mathrm{nl}}$ in Fig. 1(a)]. This was the main reason for limiting the pump power to the chosen value. Also, past this line, the dark count rate increases too much compared to the gain in the efficiency. a calibration experiment where the pump power and frequency are scanned over the relevant range of parameter space and we measure the probability $p_{e}$ to find the qubit in the excited state. We first perform a control experiment, where the buffer is left undriven [Fig. 6(a)]. The experiment is then repeated with a small coherent drive pulse on the buffer [Fig. 6(b)]. The line corresponding to the process $\hat{b} \hat{\sigma}^{\dagger} \hat{w}^{\dagger}$ is clearly identified since the qubit excitation probability $p_{e}$ vanishes in the absence of buffer drive. For each pump power, the frequency $f_{p 0}$ that maximizes the qubit excitation probability within the relevant line is represented with a red dot in Fig. 8, and it is also reported in Fig. 1(a). The linear dependence of $f_{p 0}$ as a function of $\left|\xi_{p}\right|^{2}$ is used, together with the independently measured value $\chi_{q q}=146 \mathrm{MHz}$, to calibrate $\left|\xi_{p}\right|^{2}$ in terms of photon number via the relation (G1). We attribute the spurious line appearing in both control and calibration experiments to the sixth-order nonlinear process $\xi_{p}^{3} \hat{b}^{\dagger} \sigma^{\dagger} \hat{\sigma}_{e f}^{\dagger}$, where $\hat{\sigma}_{e f}$ is the lowering operator between the second and first qubit excited states. Indeed, the frequency and slope of the line as a function of pump power are in good qualitative agreement with the expected value $\omega_{p \text {,spurious }}=\left(\bar{\omega}_{q}+\bar{\omega}_{q}^{e f}+\omega_{b}\right) / 3$.

\section{APPENDIX H: TOMOGRAPHY OF THE ITINERANT TRANSMITTED PHOTON}

The nonlinear process at the heart of the single photon detector converts the incoming photons (centered around the buffer frequency $\omega_{b}$ ) into photons emitted into the waste line at the waste frequency $\omega_{w}^{e}$ (the frequency of the waste when the qubit is in the excited state). The field propagating in the waste line is amplified by a near quantum-limited phase-preserving amplifier and demodulated at the frequency $\left(\omega_{w}^{g}+\omega_{w}^{e}\right) / 2$. Examples of demodulated time traces, with a sampling rate of $50 \mathrm{MS} / \mathrm{s}$ are shown in Fig. 7(c). Besides providing a strongly damped mode for the converted photons, in our experiment, the waste is used to read out the qubit state. Consequently, the time traces are split in two consecutive segments [see Fig. 7(c)]. The first part of the traces of duration $t_{m}=3.4 \mu$ s contains an excess noise directly attributable to the up-converted photon and are used to reconstruct the quantum state of propagating field. The second part of the traces of duration $t_{r}=0.6 \mu \mathrm{s}$, during which the waste cavity is coherently driven by the readout pulse, are used to infer the qubit state.

\section{Determination of the outgoing photon mode shape}

The complex mode shape in which single photons are emitted by the waste corresponds to the input pulse mode shape which is filtered by the buffer and waste bandwidth as well as the qubit thresholding response, and it also undergoes an overall frequency conversion. We employ the method described in Ref. [38] to determine this waveform 
(a)

(b)

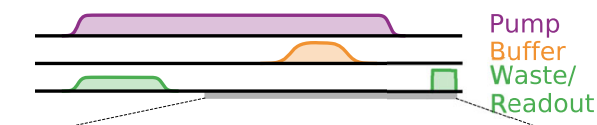

(c)
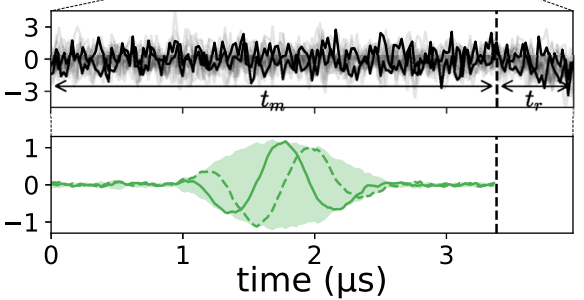

(d)
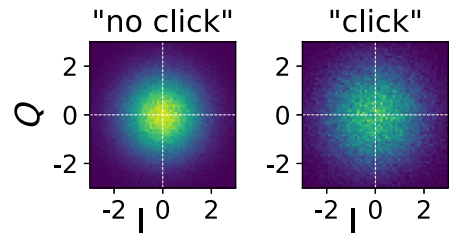

(e)
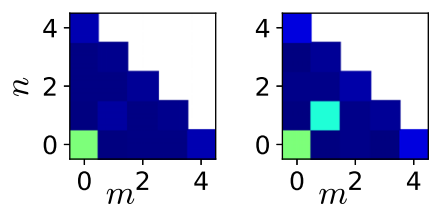

(f)

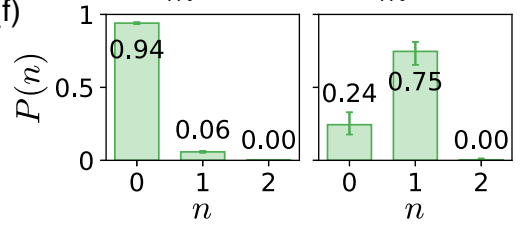

(g)

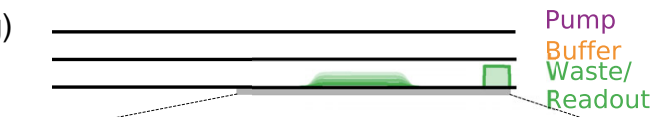

(h) 3

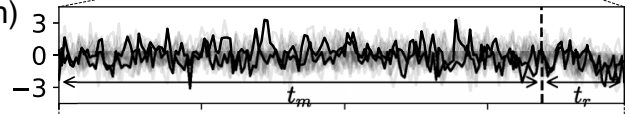

(i)
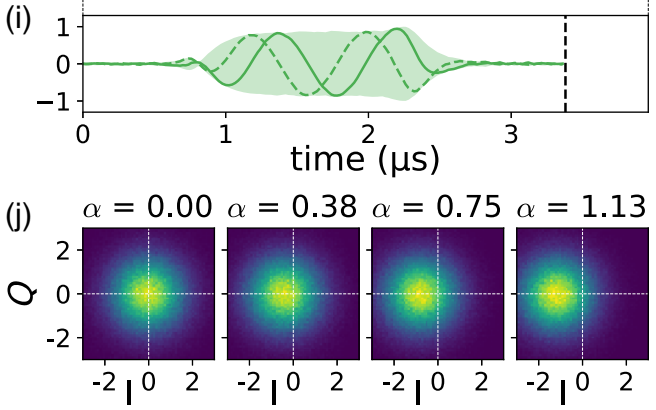

(k)

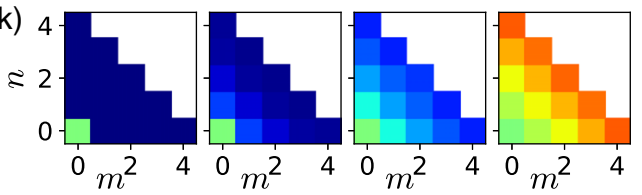

FIG. 7. Tomography of the itinerant field states. Measurement of the field emitted during a detection sequence (left) and calibration experiment (right). (a),(g) Pulse sequences for the state-reconstruction experiment (a) and amplification calibration (g). The field emitted by the waste is monitored by a phase-preserving amplifier (gray rectangle). (b),(h) Experimental traces demodulated at $\left(\omega_{w}^{g}+\omega_{w}^{e}\right) / 2$ (only one quadrature is represented): the first part of the trace (duration $t_{m}$ ) is used for the itinerant field state reconstruction while the second part (duration $t_{r}$ ) is used for qubit state discrimination. (c),(i) Mode shape determined experimentally for the single photon (c) and coherent fields (i) used for calibration. (d),(j) Measured phase space distribution of the propagating field before amplification $\hat{S} / \sqrt{G}$; it corresponds to the Husimi- $Q$ representation of the quantum state broadened by the equivalent input noise of the amplifier expressed in units of square root of photons. The distribution is conditioned on the detection of the qubit in $|g\rangle$ or $|e\rangle$ (d) and for the various coherent state amplitudes used for calibration (j). (e),(k) Experimental reconstruction of the moments of the distribution $\left\langle\left(\hat{a}^{\dagger}\right)^{n} \hat{a}^{m}\right\rangle_{\rho}$ obtained by inverting Eq. (H5). (f) Diagonal of the reconstructed density matrix. The error bars are estimated assuming a $\pm 0.5 \mathrm{~dB}$ miscalibration of the amplification chain gain.

experimentally from the 88000 traces where the qubit was detected in state $|e\rangle$ : we first combine the raw $I$ and $Q$ quadratures from the digitizer to form the complex discretetime traces:

$$
z\left(t_{j}\right)=I\left(t_{j}\right)+i Q\left(t_{j}\right)
$$

We then compute the autocorrelation matrix $\left\langle z^{*}\left(t_{j}\right) z\left(t_{k}\right)\right\rangle$ by averaging the two-time correlators over the full dataset. The autocorrelation matrix is diagonalized to find the basis of uncorrelated temporal modes. While most of the eigenvalues have a comparable weight, corresponding to the vacuum and amplifier noise in the unpopulated temporal modes, the largest eigenvalue is significantly larger (by $\sim 25 \%$ ). The corresponding complex eigenfunction $f\left(t_{j}\right)$ provides thereby the optimal temporal mode, as plotted in Figs. 4(c) and 7(c), and is normalized such that $\sum_{j}\left|f\left(t_{j}\right)\right|^{2}=1$.

\section{Photon reconstruction}

Now that the temporal shape of the itinerant mode is identified, we will focus on its quantum state. Following Ref. [39], we infer the quantum state in the mode shape $f$ by calculating the various moments of the complex amplitude distribution:

$$
S=\sum_{j} f^{*}\left(t_{j}\right) z\left(t_{j}\right)
$$

The experimental phase-space distribution of $S$ conditioned on measuring the qubit in $|g\rangle$ and $|e\rangle$ is presented in Fig. 7(d). The measured distributions-that would 
correspond to the field's $Q$ function for a quantum-limited detection - are significantly broadened by the amplifier's classical noise. However, a clear excess noise is visible on the distribution corresponding to $|e\rangle$, that reflects the increased fluctuations in the mode of interest due to the emitted photon. On a formal level, the observable $\hat{S}$ can be expressed as a function of the amplifier gain $G$, the annihilation operator $\hat{a}$ in mode $f$, and the annihilation operator $\hat{h}$ in an external mode describing the noise added by the amplifier:

$$
\hat{S}=\sqrt{G}\left(\hat{a}+\hat{h}^{\dagger}\right) .
$$

Under the assumption that the amplifier noise $\hat{h}$ is uncorrelated with the signal $\hat{a}$, we get the following expression for the moments of $\hat{S}$ [39]:

$$
\begin{aligned}
& \left\langle\left(\hat{S}^{\dagger}\right)^{n} \hat{S}^{m}\right\rangle_{\rho} \\
& =G^{(n+m) / 2} \sum_{i, j=0}^{n, m}\left(\begin{array}{c}
m \\
j
\end{array}\right)\left(\begin{array}{c}
n \\
i
\end{array}\right)\left\langle\left(\hat{a}^{\dagger}\right)^{i} \hat{a}^{j}\right\rangle_{\rho}\left\langle\hat{h}^{n-i}\left(\hat{h}^{\dagger}\right)^{m-j}\right\rangle .
\end{aligned}
$$

Remarkably, the moments $\left\langle\hat{h}^{n}\left(\hat{h}^{\dagger}\right)^{m}\right\rangle=G^{-(n+m) / 2} \times$ $\left\langle\left(\hat{S}^{\dagger}\right)^{n} \hat{S}^{m}\right\rangle_{|0\rangle\langle 0|}$ can be retrieved experimentally by performing a calibration experiment with the mode $\hat{a}$ in the vacuum state, where $\left\langle\left(\hat{S}^{\dagger}\right)^{n} \hat{S}^{m}\right\rangle_{|0\rangle\langle 0|}$ comes from the raw data and the only remaining unknown is the gain $G$.

The gain $G$ is calibrated by a control experiment. We send a well-calibrated pulse (see Ref. [36]) on the waste port which reflects back and gets detected by the digitizer [Figs. 7(f) and 7(g)]. Provided the waste is overcoupled to its transmission line (see Table I), the coherent state in the reflected mode shape [Fig. 7(h)] is equal to the incoming one. Assuming the noise is unbiased, we have $\langle\hat{S}\rangle=\sqrt{G}\langle\hat{a}\rangle$, and, hence, measuring $\langle\hat{S}\rangle$ for 3 values of $\langle\hat{a}\rangle$ [Fig. 7(i)], we deduce $G$.

The relation $(\mathrm{H} 5)$ can then be inverted to obtain the moments of the field's distribution $\left\langle\left(\hat{a}^{\dagger}\right)^{n} \hat{a}^{m}\right\rangle_{\rho_{\exp }}$, where $\rho_{\exp }$ is the experimental density matrix to be reconstructed [Figs. 7(e) and 7(j)]. We perform this reconstruction up to fourth order $(n+m \leq 4)$. We finally perform a maximumlikelihood estimation of the field's density matrix $\rho_{\mathrm{ml}}$ by minimizing the distance between the measured and expected moments:

$$
\mathcal{D}\left(\rho_{\exp }, \rho_{\mathrm{ml}}\right)=\sum_{\substack{m, n \\ m+n \leq 4}}\left|\left\langle\left(\hat{a}^{\dagger}\right)^{n} \hat{a}^{m}\right\rangle_{\rho_{\exp }}-\left\langle\left(\hat{a}^{\dagger}\right)^{n} \hat{a}^{m}\right\rangle_{\rho_{\mathrm{ml}}}\right|^{2} .
$$

The Wigner functions plotted in Fig. 4 are calculated from the density matrix $\rho_{\mathrm{ml}}$.

\section{QND-ness budget}

In this section, we estimate the fidelity expected for the observation of the Fock state $|1\rangle$ out of the waste mode conditioned on the click of the detector as depicted in Fig. 4 as well as the fidelity expected for the observation of the Fock state $|1\rangle$ out of the waste mode conditioned on the click of the detector. This calculation aims at taking into account the main imperfections of the detector: the readout infidelity, the dark counts originating from qubit thermal excitations, the finite detection efficiency, and the photon statistics arising from the coherent state. The contribution

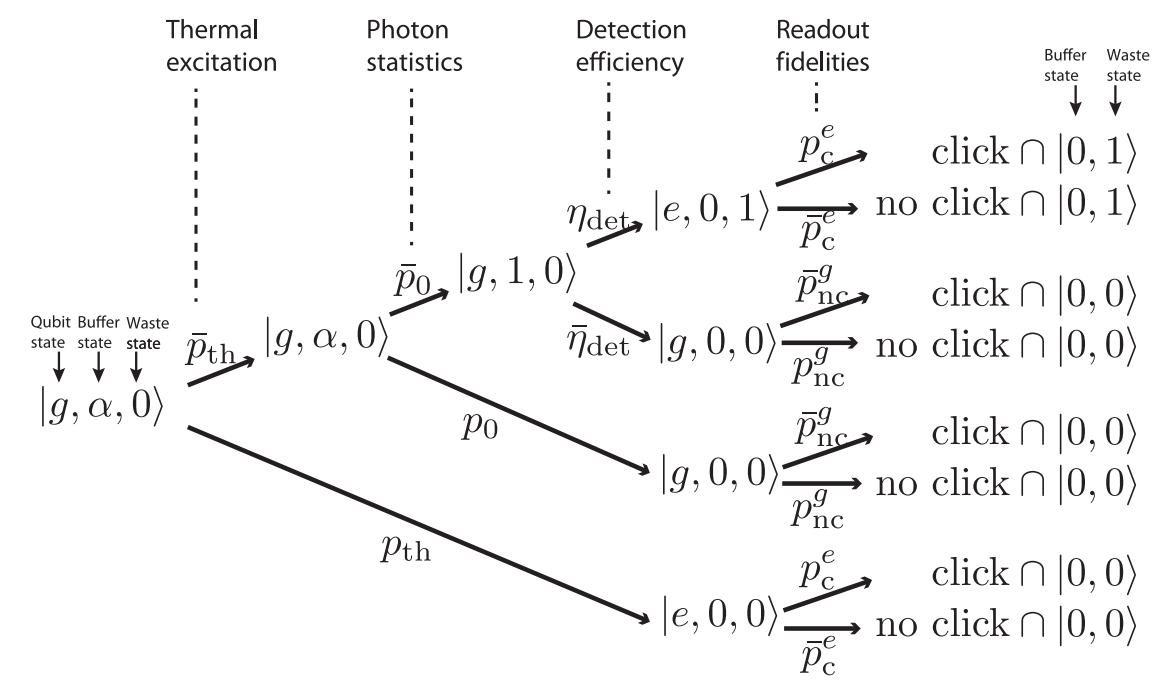

FIG. 8. Probability tree for modeling imperfection of the detector and quantum nondemolition character of the device. The input of the tree denotes the joint state of the qubit, the buffer, and the waste, respectively. Starting from the qubit in the ground state and the input field in a coherent state $\alpha$, a spurious thermal excitation can flip the qubit with a probability $p_{\text {th }}$, the coherent state gets projected to the vacuum state with a probability $p_{0}$, the photon gets detected with a probability $\eta_{\text {det }}$, and the qubit gets read out correctly with a probability $p_{\mathrm{c}}^{e}$ when in its excited state or $p_{\mathrm{nc}}^{g}$ when in its ground state. 
of each imperfection can be quantified separately and summarized on an event tree shown in Fig. 8.

(i) Thermal excitation. The probability of observing a thermal excitation caused by the thermal environment and the pump heating due to the waiting time $t_{\text {wait }, 1}=3 \mu$ s between the reset and the photon arrival is given by $p_{\text {th }}=1-\exp \left(-\Gamma_{\mathrm{dc}} t_{\mathrm{wait}, 1}\right)+0.003=$ 0.0149 .

(ii) Photon statistics. The probability of being in the vacuum state with an input coherent state of mean photon number $\bar{n}=0.35$ is given by $p_{0}=$ $\exp (-\bar{n})=0.70$.

(iii) Detection inefficiency. The detection efficiency for a $1-\mu$ s-long pulse discarding the readout infidelity is expected to be $\eta_{\text {det }}=0.58 / 0.94=0.62$ according to Fig. 2.

(iv) Readout infidelity. The qubit readout fidelities can be estimated precisely including the waiting time between the photon arrival and readout pulse of $t_{\text {wait }, 2}=3 \mu \mathrm{s}$. Taking into account the relaxation time $T_{1}$ and the residual thermal heating $\Gamma_{\mathrm{dc}}=T_{1} n_{\mathrm{th}}$, it is given by $p_{\mathrm{c}}^{e}=0.94 \exp \left(-t_{\text {wait }, 2} / T_{1}\right)=0.72$ and $p_{\mathrm{nc}}^{g}=0.997 \exp \left(-\Gamma_{\mathrm{dc}} t_{\mathrm{wait}, 2}\right)=0.98, \quad$ respectively, the probability of clicking (not clicking) while the qubit is in its excited (ground) state.

We build an event tree in order to model the contribution of each inefficiency; this event tree is depicted in Fig. 8. Starting from the qubit in its ground state and a coherent state in the input line, each source of inefficiency, as listed above, leads to the occurrence probability of each branch. Using this tree, we compute the probability of having a click $p$ (click) using the notation $\bar{p}=1-p$.

$$
\begin{aligned}
p(\text { click }) & =\sum_{\text {branches }} p(\text { click } \cap|0,0\rangle)+p(\text { click } \cap|0,1\rangle) \\
& =\bar{p}_{\mathrm{th}}\left[p_{0} \bar{p}_{\mathrm{nc}}^{g}+\bar{p}_{0}\left(\bar{\eta}_{\mathrm{det}} \bar{p}_{\mathrm{nc}}^{g}+\eta_{\mathrm{det}} p_{\mathrm{c}}^{e}\right)\right]+p_{\mathrm{th}} p_{\mathrm{c}}^{e} \\
& =0.14
\end{aligned}
$$

Then, we compute the probability of observing the Fock state $|1\rangle$ going out of the waste mode conditioned on the click of the detector using standard conditional probability relations:

$$
\begin{aligned}
p(|1\rangle \mid \text { click }) & =\frac{p(\text { click } \cap|1\rangle)}{p(\text { click })} \\
& =\frac{\bar{p}_{\text {th }} \bar{p}_{0} \eta_{\text {det }} p_{\mathrm{c}}^{e}}{\bar{p}_{\text {th }}\left[p_{0} \bar{p}_{\mathrm{nc}}^{g}+\bar{p}_{0}\left(\bar{\eta}_{\mathrm{det}} \bar{p}_{\mathrm{nc}}^{g}+\eta_{\operatorname{det}} p_{\mathrm{c}}^{e}\right]+p_{\mathrm{th}} p_{\mathrm{c}}^{e}\right.} \\
& =0.80, \\
p(|1\rangle \mid \text { no click }) & =\frac{p(\text { no click } \cap|1\rangle)}{1-p(\text { click })} \\
& =\frac{\bar{p}_{\text {th }} \bar{p}_{0} \eta_{\mathrm{det}} \bar{p}_{\mathrm{c}}^{e}}{1-\left\{\bar{p}_{\mathrm{th}}\left[p_{0} \bar{p}_{\mathrm{nc}}^{g}+\bar{p}_{0}\left(\bar{\eta}_{\mathrm{det}} \bar{p}_{\mathrm{nc}}^{g}+\eta_{\mathrm{det}} p_{\mathrm{c}}^{e}\right)\right]+p_{\text {th }} p_{\mathrm{c}}^{e}\right\}} \\
& =0.047 .
\end{aligned}
$$

These probabilities are directly related to the QND-ness presented in Fig. 4, in particular: $P_{|0\rangle}^{\text {no click }}=1-P_{|1\rangle}^{\text {no click }}=$ 0.95 and $P_{|1\rangle}^{\text {click }}=p(|1\rangle \mid$ click $)=0.80$. On the one hand, the vacuum fidelity conditioned on the absence of a click $P_{|0\rangle}^{\text {no click }}$ agrees well with the experimental fidelity (0.94). On the other hand, the Fock state $|1\rangle$ fidelity conditioned on a click of the detector $P_{|1\rangle}^{\text {click }}$ is significantly larger than the experimental value (0.75). This discrepancy can be attributed to two experimental imperfections.

(i) Moment reconstruction. The reconstruction of the density matrix from heterodyne detection is based on the measurement of high-order moments (up to fourth order). The fourth-order moment of the reconstruction $\left\langle\hat{S}^{\dagger 4}\right\rangle_{\rho}$ is unexpectedly large.

The signal coming out from the waste mode cannot be linked to any reference oscillator phase $\phi_{w}$. Indeed, the phases of signals are constrained through the parametric conversion by the following phase relation: $\phi_{p}=\phi_{q}+\phi_{w}-\phi_{b}$. The only oscillator phases available are the pump oscillator phase $\phi_{p}$ and the input photon oscillator phase $\phi_{b}$. However, during the conversion process, there is no reference oscillator associated to the qubit; therefore $\phi_{q}$ remains undefined. As a consequence, the phase of the demodulation oscillator $\phi_{w}$ must be arbitrary, and the off-diagonal component should vanish.

The nonzero contribution of off-diagonal moments is attributed to statistical fluctuations due to limited dataset. The effect of this spurious higherorder moment can be visualized as the slight quadrupole component in the Wigner distribution shown in Fig. 4. In order to emphasize this point, we have performed an error analysis of the moment 
reconstruction from the dataset based on the bootstrap method [42]. The result of this analysis is shown as confidence interval in the moment matrix written in Eq. (H9). We observed significant uncertainties for the off-diagonal components as expected. It clearly indicates that the quadrupole components of the state reconstruction can be attributed to statistical fluctuations.

$$
\left|\left\langle\left(\hat{a}^{\dagger}\right)^{n} \hat{a}^{m}\right\rangle_{\rho_{\text {photon }}}\right|=\left(\begin{array}{ccccc}
1.00 \pm 0.00 & 0.00 \pm 0.01 & 0.02 \pm 0.01 & 0.01 \pm 0.04 & 0.17 \pm 0.15 \\
0.00 \pm 0.01 & 0.78 \pm 0.01 & 0.02 \pm 0.03 & 0.04 \pm 0.07 & X \\
0.02 \pm 0.01 & 0.02 \pm 0.03 & 0.07 \pm 0.06 & X & X \\
0.01 \pm 0.04 & 0.04 \pm 0.07 & X & X & X \\
0.17 \pm 0.15 & X & X & X & X
\end{array}\right) .
$$

A reasonable assumption is to consider the distribution to be phase invariant since there is no absolute local oscillator associated with the converted signal. Indeed, during the conversion process, the phase of the incoming photon is mixed with the pump phase and the qubit phase. The latter is not referenced at any point since the qubit sits in either its ground state or its excited state (no defined phases are associated to these states).

If we assume the distribution to be phase invariant (i.e., the off-diagonal element to be zero), then the probability of finding Fock state $|1\rangle$ is improved to $P_{|1\rangle}^{\text {click }}=0.78$.

(ii) Calibration of amplifying chain gain. Finally, we suspect a slight uncertainty on the gain of the amplifying chain in the vicinity of the waste mode.

The waste photon is released at a frequency $\omega_{w}-$ $\chi_{q w}$ since the qubit is its excited states. However, the calibrations have been performed at a frequency $\omega_{w}$ since the qubit was in its ground state. This slight shift in frequency of $\chi_{q w} / 2 \pi=2.73 \mathrm{MHz}$ can lead to a slight discrepancy in the gain of the amplifying chain, which is usually negligible since the electromagnetic environment is often considered as flat over such a small scale.
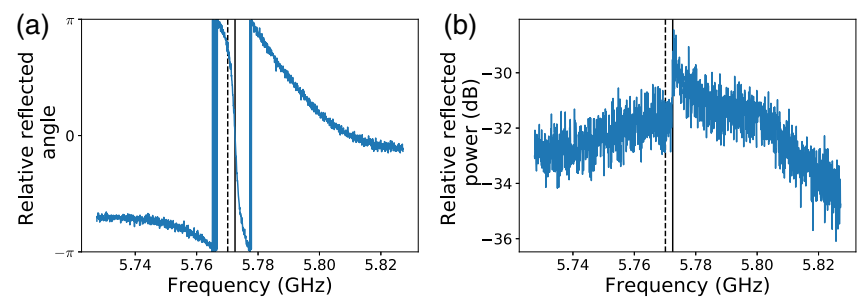

FIG. 9. Spectroscopy of the waste mode. (a),(b) Relative reflected phase (a) and power (b) ( $y$ axis) of a small spectroscopic tone on the waste port for varying frequencies ( $x$ axis). (a) The sharp $2 \pi$ phase roll corresponds to the waste mode, whereas the wide one corresponds to the Purcell filter. (b) Because of the presence of a lossy Purcell filter (large interdigitated capacitor; see Fig. 5), the relative reflected power is not flat around the waste frequency (solid line). The detected photon comes out of the detector with $\chi_{q w}$ frequency shift (dashed line).
However, due to the presence of the resonant Purcell filter at the waste frequency shown in Fig. 5, we observed some significant variation of the amplitude of reflection coefficient on scales of order $\chi_{q w}$ as shown in Fig. 9. An uncertainty of the total gain of the amplifying chain of order $\pm 0.5 \mathrm{~dB}$ can be attributed to this resonant structure.

[1] R. H. Hadfield, Single-Photon Detectors for Optical Quantum Information Applications, Nat. Photonics 3, 696 (2009).

[2] A. Ourjoumtsev, R. Tualle-Brouri, J. Laurat, and P. Grangier, Generating Optical Schrödinger Kittens for Quantum Information Processing, Science 312 (2006).

[3] P. C. Humphreys, N. Kalb, J. P. Morits, R. N. Schouten, R. F. Vermeulen, D. J. Twitchen, M. Markham, and R. Hanson, Deterministic Delivery of Remote Entanglement on a Quantum Network, Nature (London) 558, 268 (2018).

[4] S.-K. Liao et al., Satellite-Relayed Intercontinental Quantum Network, Phys. Rev. Lett. 120, 030501 (2018).

[5] R. Raussendorf and H. J. Briegel, A One-Way Quantum Computer, Phys. Rev. Lett. 86, 5188 (2001).

[6] C. Monroe, R. Raussendorf, A. Ruthven, K. R. Brown, P. Maunz, L.-M. Duan, and J. Kim, Large-Scale Modular Quantum-Computer Architecture with Atomic Memory and Photonic Interconnects, Phys. Rev. A 89, 022317 (2014).

[7] X. Gu, A. F. Kockum, A. Miranowicz, Y. xi Liu, and F. Nori, Microwave Photonics with Superconducting Quantum Circuits, Phys. Rep. 718-719, 1 (2017).

[8] F. Helmer, M. Mariantoni, E. Solano, and F. Marquardt, Quantum Nondemolition Photon Detection in Circuit QED and the Quantum Zeno Effect, Phys. Rev. A 79, 052115 (2009).

[9] G. Romero, J. J. García-Ripoll, and E. Solano, Microwave Photon Detector in Circuit QED, Phys. Rev. Lett. 102, 173602 (2009).

[10] S. R. Sathyamoorthy, L. Tornberg, A. F. Kockum, B. Q. Baragiola, J. Combes, C. M. Wilson, T. M. Stace, and G. Johansson, Quantum Nondemolition Detection of a Propagating Microwave Photon, Phys. Rev. Lett. 112, 093601 (2014). 
[11] J. Leppäkangas, M. Marthaler, D. Hazra, S. Jebari, R. Albert, F. Blanchet, G. Johansson, and M. Hofheinz, Multiplying and Detecting Propagating Microwave Photons Using Inelastic Cooper-Pair Tunneling, Phys. Rev. A 97, 013855 (2018).

[12] B. Royer, A. L. Grimsmo, A. Choquette-Poitevin, and A. Blais, Itinerant Microwave Photon Detector, Phys. Rev. Lett. 120, 203602 (2018).

[13] Y.-F. Chen, D. Hover, S. Sendelbach, L. Maurer, S. T. Merkel, E. J. Pritchett, F. K. Wilhelm, and R. McDermott, Microwave Photon Counter Based on Josephson Junctions, Phys. Rev. Lett. 107, 217401 (2011).

[14] A. Narla, S. Shankar, M. Hatridge, Z. Leghtas, K. M. Sliwa, E. Zalys-Geller, S. O. Mundhada, W. Pfaff, L. Frunzio, R. J. Schoelkopf, and M. H. Devoret, Robust Concurrent Remote Entanglement between Two Superconducting Qubits, Phys. Rev. X 6, 031036 (2016).

[15] A. Opremcak, I. V. Pechenezhskiy, C. Howington, B. G. Christensen, M. A. Beck, E. Leonard, J. Suttle, C. Wilen, K. N. Nesterov, G. J. Ribeill, T. Thorbeck, F. Schlenker, M. G. Vavilov, B. L. T. Plourde, and R. McDermott, Measurement of a Superconducting Qubit with a Microwave Photon Counter, Science 361, 1239 (2018).

[16] S. Kono, K. Koshino, Y. Tabuchi, A. Noguchi, and Y. Nakamura, Quantum Non-Demolition Detection of an Itinerant Microwave Photon, Nat. Phys. 14, 546 (2018).

[17] J.-C. Besse, S. Gasparinetti, M. C. Collodo, T. Walter, P. Kurpiers, M. Pechal, C. Eichler, and A. Wallraff, SingleShot Quantum Nondemolition Detection of Individual Itinerant Microwave Photons, Phys. Rev. X 8, 021003 (2018).

[18] H. J. Kimble, The Quantum Internet, Nature (London) 453, 1023 (2008).

[19] S. K. Lamoreaux, K. A. van Bibber, K. W. Lehnert, and G. Carosi, Analysis of Single-Photon and Linear Amplifier Detectors for Microwave Cavity Dark Matter Axion Searches, Phys. Rev. D 88, 035020 (2013).

[20] P. Haikka, Y. Kubo, A. Bienfait, P. Bertet, and K. Mølmer, Proposal for Detecting a Single Electron Spin in a Microwave Resonator, Phys. Rev. A 95, 022306 (2017).

[21] P. A. Morris, R. S. Aspden, J. E. Bell, R. W. Boyd, and M. J. Padgett, Imaging with a Small Number of Photons, Nat. Commun. 6, 5913 (2015).

[22] G. Nogues, A. Rauschenbeutel, S. Osnaghi, M. Brune, J. Raimond, and S. Haroche, Seeing a Single Photon without Destroying It, Nature (London) 400, 239 (1999).

[23] S. Gleyzes, S. Kuhr, C. Guerlin, J. Bernu, S. Deléglise, U. Busk Hoff, M. Brune, J.-M. Raimond, and S. Haroche, Quantum Jumps of Light Recording the Birth and Death of a Photon in a Cavity, Nature (London) 446, 297-300 (2007).

[24] D. Schuster, A. A. Houck, J. Schreier, A. Wallraff, J. Gambetta, A. Blais, L. Frunzio, J. Majer, B. Johnson, M. Devoret et al., Resolving Photon Number States in a Superconducting Circuit, Nature (London) 445, 515 (2007).

[25] K. Inomata, Z. Lin, K. Koshino, W. D. Oliver, J.-S. Tsai, T. Yamamoto, and Y. Nakamura, Single Microwave-Photon Detector Using an Artificial $\Lambda$-Type Three-Level System, Nat. Commun. 7, 12303 (2016).
[26] E. Kapit, The Upside of Noise: Engineered Dissipation as a Resource in Superconducting Circuits, Quant. Sci. Technol. 2, 033002 (2017).

[27] J. D. Teufel, T. Donner, D. Li, J. W. Harlow, M. Allman, K. Cicak, A. J. Sirois, J. D. Whittaker, K. W. Lehnert, and R. W. Simmonds, Sideband Cooling of Micromechanical Motion to the Quantum Ground State, Nature (London) 475, 359 (2011).

[28] K. W. Murch, U. Vool, D. Zhou, S. J. Weber, S. M. Girvin, and I. Siddiqi, Cavity-Assisted Quantum Bath Engineering, Phys. Rev. Lett. 109, 183602 (2012).

[29] S. Shankar, M. Hatridge, Z. Leghtas, K. M. Sliwa, A. Narla, U. Vool, S. M. Girvin, L. Frunzio, M. Mirrahimi, and M. H. Devoret, Autonomously Stabilized Entanglement between Two Superconducting Quantum Bits, Nature (London) 504, 419 (2013).

[30] Z. Leghtas, S. Touzard, I. M. Pop, A. Kou, B. Vlastakis, A. Petrenko, K. M. Sliwa, A. Narla, S. Shankar, M. J. Hatridge, M. Reagor, L. Frunzio, R. J. Schoelkopf, M. Mirrahimi, and M. H. Devoret, Confining the State of Light to a Quantum Manifold by Engineered Two-Photon Loss, Science 347, 853 (2015).

[31] K. M. Sliwa, M. Hatridge, A. Narla, S. Shankar, L. Frunzio, R. J. Schoelkopf, and M.H. Devoret, Reconfigurable Josephson Circulator/Directional Amplifier, Phys. Rev. X 5, 041020 (2015).

[32] A. Metelmann and A. A. Clerk, Nonreciprocal Photon Transmission and Amplification via Reservoir Engineering, Phys. Rev. X 5, 021025 (2015).

[33] C. Macklin, K. O’Brien, D. Hover, M. E. Schwartz, V. Bolkhovsky, X. Zhang, W. D. Oliver, and I. Siddiqi, A Near-Quantum-Limited Josephson Traveling-Wave Parametric Amplifier, Science 350, 307 (2015).

[34] G. Kirchmair, B. Vlastakis, Z. Leghtas, S. E. Nigg, H. Paik, E. Ginossar, M. Mirrahimi, L. Frunzio, S. M. Girvin, and R. J. Schoelkopf, Observation of Quantum State Collapse and Revival due to the Single-Photon Kerr Effect, Nature (London) 495, 205 (2013).

[35] P. Krantz, A. Bengtsson, M. Simoen, S. Gustavsson, V. Shumeiko, W. D. Oliver, C. M. Wilson, P. Delsing, and J. Bylander, Single-Shot Read-Out of a Superconducting Qubit Using a Josephson Parametric Oscillator, Nat. Commun. 7, 11417 (2016).

[36] R. Lescanne, L. Verney, Q. Ficheux, M. H. Devoret, B. Huard, M. Mirrahimi, and Z. Leghtas, Escape of a Driven Quantum Josephson Circuit into Unconfined States, Phys. Rev. Applied 11, 014030 (2019).

[37] L. Verney, R. Lescanne, M. H. Devoret, Z. Leghtas, and M. Mirrahimi, Structural Instability of Driven Josephson Circuits Prevented by an Inductive Shunt, Phys. Rev. Applied 11, 024003 (2019).

[38] O. Morin, C. Fabre, and J. Laurat, Experimentally Accessing the Optimal Temporal Mode of Traveling Quantum Light States, Phys. Rev. Lett. 111, 213602 (2013).

[39] C. Eichler, D. Bozyigit, C. Lang, L. Steffen, J. Fink, and A. Wallraff, Experimental State Tomography of Itinerant Single Microwave Photons, Phys. Rev. Lett. 106, 220503 (2011). 
[40] E. A. Sete, J. M. Martinis, and A. N. Korotkov, Quantum Theory of a Bandpass Purcell Filter for Qubit Readout, Phys. Rev. A 92, 012325 (2015).

[41] S. Touzard, A. Kou, N. E. Frattini, V. V. Sivak, S. Puri, A. Grimm, L. Frunzio, S. Shankar, and M. H. Devoret,
Gated Conditional Displacement Readout of Superconducting Qubits, Phys. Rev. Lett. 122, 080502 (2019).

[42] B. Efron and R. J. Tibshirani, An Introduction to the Bootstrap (CRC Press, Boca Raton, FL, 1994). 